\title{
Prediction of installed jet noise
}

\author{
B. $\mathbf{L y u} \mathbf{u}^{1} \dagger$ \\ A. P. Dowling ${ }^{1}$ and I. Naqavi ${ }^{1}$ \\ ${ }^{1}$ Department of Engineering, University of Cambridge, Cambridge CB2 1PZ, UK \\ (Received ?; revised ?; accepted ?. - To be entered by editorial office)
}

A semi-analytical model for installed jet noise is proposed in this paper. We argue and conclude that there exist two distinct sound source mechanisms for installed jet noise and the model is therefore composed of two parts to account for these different sound source mechanisms. Lighthill's acoustic analogy and a fourth-order space-time correlation model for Lighthill stress tensor are used to model the sound induced by the equivalent turbulent quadrupole sources, while the trailing-edge scattering of near-field evanescent instability waves are modelled using Amiet's approach. A non-zero ambient mean flow is taken into account. It is found that, when the rigid surface is not so close to the jet as to affect the turbulent flow field, the trailing-edge scattering of near-field evanescent waves dominates the low frequency amplification of installed jet noise in the far-field. The high-frequency noise enhancement on the reflected side is due to the surface reflection effect. The model agrees well with experimental results at different observer angles apart from deviations caused by mean-flow refraction effect at high frequencies at low observer angles.

\section{Introduction}

Aircraft noise is believed to be one of the most commonly reported residential disturbances (Pepper et al. 2003). Among the different noise sources, jet noise is found to be the dominant component at takeoff. For the past few decades, jet noise has been one of the most heavily investigated subjects and the outcome has been quite successful. However it is worth noting that the vast majority of research work during this time is for an isolated nozzle and jet. But the realistic configuration of a modern aircraft often involves the jet engines installed below the aircraft wings, as shown in figure 1. The effects of wings and other high-lift devices on the jet noise are often referred to as jet installation effects and the resulting jet is commonly called an installed jet and compared to the isolated jet. Research has shown that the presence of solid boundaries can greatly affect acoustic source behaviours (Curle 1955; Williams \& Hall 1970). Consequently, the close presence of aircraft wings can significantly alter the jet noise heard in the far-field (Bushell 1975; Fisher et al. 1977; Way \& Turner 1980; Shearin 1983). The investigation into the installed jet noise has been, however, rather limited. Particularly, there is a general lack of reliable prediction models and also of understanding of the noise modification mechanism. This paper aims to contribute to bridging that gap: to develop a reliable model that can predict the installed jet noise and to advance the understanding of the noise amplification mechanism.

For the configuration shown in figure 1, there are two important geometric parameters defining the position of the engine relative to the wing: the distance $H$ between the jet centre line and the wing surface and the distance $L$ between jet nozzle and the trailing edge of the flap. As far as the installed jet noise is concerned, one expects the jet

$\dagger$ Email address for correspondence: bl362@cam.ac.uk 


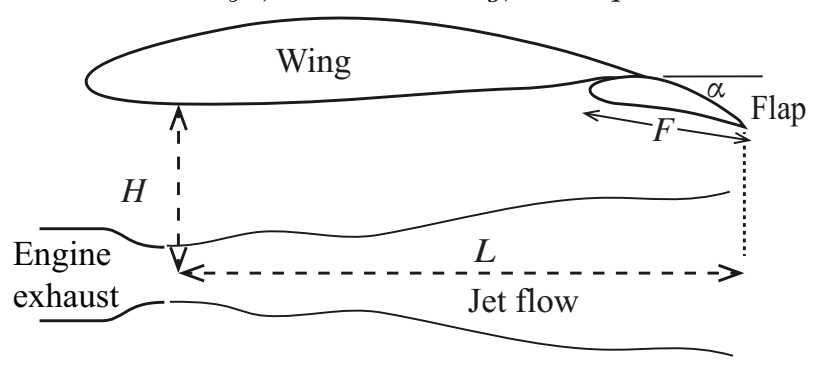

Figure 1. The schematic illustration of an installed engine. The distance between the jet centreline and the under surface of the wing is denoted by $H$ while the axial distance between the nozzle exit and the trailing edge of the flap is denoted by $L$.

operating condition to have an important impact on the sound radiated to the far-field, as described by the jet nozzle diameter $D$, the jet exit velocity $V$, the jet spread rate $\beta$ and the temperature ratio $T_{r}$ in the case of hot jets. The impact of varying $H$ and $L$ on the far-field noise will be referred to as the engine position effect, while that of varying $D, V, \beta$ will be called the jet condition effect in this paper. When the flap is deployed, the flap length $F$, the deflected angle $\alpha$ and the width $W$ of flap cut-out in a real flight vehicle have influences on the installed jet noise, and this is similarly given the name the flap position effect.

The extra noise produced by an installed engine was first noticed by Bushell (1975), who compared the in-flight installed jet noise with the static isolated jet noise for a fullscale aircraft. Many investigations followed and they fall roughly into three categories: identifying installed noise sources by studying the acoustic characteristics of an installed jet, the engine position, jet condition and flap position effects; developing installed jet noise prediction models; and investigating noise reduction techniques. The first category includes the experimental work of Head \& Fisher (1976), Szewczyk (1979), Way \& Turner (1980), Shearin (1983), Mead \& Strange (1998) and Brown (2013). Through their experimental work, it was found that increasing $H$ results in less noise at low frequencies and decreasing $L$ follows the same trend and shifts the peak frequency to higher frequencies, the velocity dependence of the peak of the low frequency augmentation is to the five to sixth power. It was also confirmed that the low frequency noise enhancement has a dipole-like directivity pattern. The numerical work carried out by Bondarenko et al. (2012) also falls into the first category and sheds more light on the understanding of installed jet via flow field visualization.

In conjunction with the above experimental and numerical studies of installed jet noise characteristics, attempts to develop prediction models for installed jet noise have also been made. These include the work by Stevens et al. (1983), Sengupta (1983), Moore (2004) and Bhat \& Blackner (1998). With the exception of Moore's model, which is based on 3D ray theory and jet blockage profiles and gives encouraging results at high frequencies, the prediction models proposed at this time were generally empirical, i.e. by fitting experimental data or superimposing parametric changes heuristically. Thus these models were inherently unable to capture detailed characteristics of installed jet noise correctly or to enhance physical understanding of the mechanisms. However, very recently some less-empirical models were proposed, including the work of Vera et al. (2015) and Piantanida et al. (2015). In Vera's work, for an assumed plane-wave-like incident field the scattered pressure on the surface of a semi-infinite flat plate is obtained by making use of Wiener-Hopf method and the far-field sound is obtained using Amiet's approach. However the proposed form of the statistical spectrum of "the incident field required to 
calculate the far-field sound is hard to obtain in experimental measurements". In addition the ambient flow (non-trivial in a real flight certification process) is neglected in their modelling. Piantanida et al. (2015) adopted another approach in their work, where the half-plane scattering Green's function and an inferred near-field pressure source of the wave-packet form were used. The far-field sound is obtained by performing numerical integration. Both experimental measurements and numerical model predictions for low frequency installed jet noise were presented, and "a good overall agreement with the experiments in terms of the dependence of the radiated levels and directivity on the radial jet-plate separation and sweep angle" is achieved. In Piantanida's work a Boundary Element Method calculation is also performed as a validation for the Green's function approach. The same technique was used earlier by Papamoschou (2010) to predict the jet noise shielding effects with an inferred wavepackage and a monopole as sources. Reasonable results were obtained with limitations at high frequencies caused by the deterministic characteristics of the sound sources. Attempts trying to reduce the installation effects of jet noise, as characterised by the third category, were first made by Wang (1981). In his paper, Wang reported an experimental test of jet noise performed on wing models made of materials with different acoustic surface properties. The experimental results showed that the wing models with specially treated surfaces can substantially reduce the noise enhancement at high frequencies. Of practical interest, it was mentioned by Piantanida et al. (2015) that if the wing is swept, the installed jet noise can be reduced effectively, and the larger the swept angle is, the more the sound is reduced.

Hitherto, there has been little doubt about the mechanism of installation effect at high frequencies: the noise is generated by the small scale eddies in the jet and reflected off the wing and flap surface; the reflected noise is refracted and attenuated by the presence of the turbulent jet plume. On the other hand, the noise mechanism at low frequencies has not been accepted unanimously. One of the proposed ideas was that the noise is due to the trailing-edge scattering, while Shearin (1983) suggested a jet-surface interaction mechanism and Pastouchenko \& Tam (2007) argued that it is the downwash effect of the wing flap causing more turbulence in the jet that is the principal mechanism. However, examination of the acoustic properties of the low frequency installed noise and its dependence on the engine position, jet condition and flap position leads one to believe that the dominant effect is the trailing-edge noise. This is because the dipole characteristics are in agreement with the observed directivity pattern of trailing-edge noise at low frequencies (Head \& Fisher 1976; Wang 1981; Mead \& Strange 1998; Bondarenko et al. 2012; Brown 2013; Amiet 1976b; Roger \& Moreau 2005), decreasing $L$ results in a reduced noise and a frequency shift toward high frequencies (Head \& Fisher 1976; Way \& Turner 1980; Stevens et al. 1983; Wang 1981; Shearin 1983), there exists a high correlation between the pressure field near the trailing edge and the far-field sound (Head \& Fisher 1976) and the dependence of sound intensity on the characteristic jet velocity is to the 5 to 6 power (Head \& Fisher 1976; Brown 2013). These trailing-edge noise characteristics remind us the suitablity of Amiet's approach in modelling the low-frequency installed jet noise.

In the next section we present the detailed derivation of our hybrid model. Section 3 then presents results based on the new model, and comparison with experimental data is also shown. The last section gives a brief conclusion of this paper.

\section{Model formulation}

Acoustic analogy theories have long been the standard approaches to predict jet noise and they have proved to be sufficient in predicting isolated jet noise Lighthill (1952); 


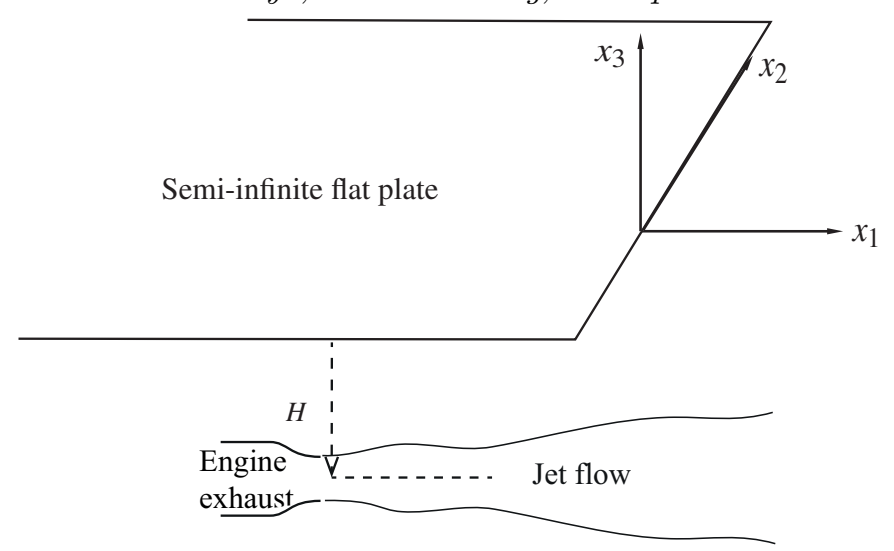

Figure 2. Schematic of the simplified model with a semi-infinite flat plate.

Williams (1963); Lilley (1974); Goldstein (2003). We attempt to adopt the same approaches to the modelling of installed jet noise. However, as we will see in subsequent sections, conventional acoustic analogy theories fail to capture an additional but important noise generation mechanism. Consequently, in this section we use Lighthill's acoustic analogy theory in conjunction with an additional near-field scattering theory to predict the far-field sound of installed cold jets at low Mach numbers, which naturally divides this section into two parts.

\subsection{Lighthill's acoustic analogy}

In Lighthill's original work (Lighthill 1952, 1954), turbulence generated noise was studied in the absence of any solid boundaries. The effect of boundaries on sound generation aerodynamically was investigated by Curle (1955). Curle's theory states that the effect of a solid boundary is equivalent to a distribution of dipole sources (unsteady force source) in addition to the Lighthill's quadrupole sources. The dipole strength is equal to the surface pressure. Therefore in order to use Curle's approach to calculate far-field sound, the pressure on the solid boundaries has to be known beforehand. The pressure on the surface of the plate depends on the quadrupole sources and is difficult to obtain when the surface is non-compact, where there may be significant phase cancellation of the sound from the dipole sources. An alternative approach, which is much more advantageous, is that one can use the Green's function that has satisfies the boundary conditions on the solid surfaces. Then, the far-field sound, including both the incident field due to the quadrupole sources and the scattered field due to solid boundaries, can be readily obtained by performing a volume integration of the product of the Green's function and the quadrupole sources. This is the approach that we use in this paper.

To use the latter approach, the Green's function satisfying the rigid-wall boundary conditions at the upper and lower surfaces of the aircraft wing and flap needs to be obtained. While this could be done numerically by, for example, boundary element method, we seek an analytical solution so that the physics of the jet-wing interaction can be understood. Therefore we simplify the geometry by replacing the wing-flap system with a semi-infinite flat plate, as shown in figure 2. This is believed to be valid especially when the acoustic wavelength is shorter than the wing size (Amiet 1976b; Roger \& Moreau 2005). Then we start with the equation obtained by Lighthill (Lighthill 1952), i.e.

$$
\left(\frac{\partial^{2}}{\partial t^{2}}-c_{0}^{2} \nabla^{2}\right)\left(\rho-\rho_{0}\right)=\frac{\partial^{2} T_{i j}}{\partial x_{i} \partial x_{j}},
$$


where $x_{i}(i=1,2,3)$ denote the Cartesian coordinates shown in figure $2, \rho$ the fluid density, $\rho_{0}$ the ambient fluid density, $c_{0}$ the speed of sound in the ambient fluid, and the Lighthill's stress tensor has the form of

$$
T_{i j}=\rho u_{i} u_{j}+p_{i j}-\left(\rho-\rho_{0}\right) c_{0}^{2} \delta_{i j}
$$

where $u_{i}$ and $u_{j}$ are the velocity components in the $i$ and $j$ direction respectively, $p_{i j}$ the stress tensor. When Reynolds number is high, which is so for most industrially relevant jet flows (and for many laboratory jets), the viscous terms in $T_{i j}$ can be ignored (Lighthill 1952; Goldstein 2003; Karabasov et al. 2010). Also when the mean temperature of the jet is same as that of the ambient fluid, which is a good approximation for cold jets at low Mach numbers, the assumption that fluctuations in pressure is balanced out by the product of the density fluctuations and $c_{0}^{2}$ can be made (Lighthill 1952). $T_{i j}$ can thus be approximated by

$$
T_{i j} \approx \rho u_{i} u_{j}
$$

In realistic full-scale tests, there exists a uniform ambient flow $U$ in $x_{1}$ direction due to the forward flight of the aircraft. We aim to include this ambient mean flow effect in our model, and to do that it is convenient to express the fluid velocity in terms of the fluctuation velocity (relative to the background flow) $u_{j}^{b}$, i.e. $u_{i}=u_{i}^{b}+U \delta_{i 1}$. Substituting this definition into (2.1) and making use of the mass conservation equation, (2.1) can be formulated as

$$
\left(\frac{\partial}{\partial t}+U \frac{\partial}{\partial x_{1}}\right)^{2} \rho^{\prime}-c_{0}^{2} \nabla^{2} \rho^{\prime}=\frac{\partial^{2} \rho u_{i}^{b} u_{j}^{b}}{\partial x_{i} \partial x_{j}},
$$

where we define the density fluctuation $\rho^{\prime} \equiv \rho-\rho_{0}$. By assuming a $\mathrm{e}^{\mathrm{i} \omega t}$ time dependence, (2.3) can be written as

$$
\left(\mathrm{i} \omega+U \frac{\partial}{\partial x_{1}}\right)^{2} \rho^{\prime}(\boldsymbol{x}, \omega)-c_{0}^{2} \nabla^{2} \rho^{\prime}(\boldsymbol{x}, \omega)=\frac{\partial^{2} \hat{T}_{i j}(\boldsymbol{x}, \omega)}{\partial x_{i} \partial x_{j}} .
$$

where $\rho^{\prime}(\boldsymbol{x}, \omega)$ and $\hat{T}_{i j}(\boldsymbol{y}, \omega)$ are the Fourier transformations of $\rho^{\prime}(\boldsymbol{x}, t)$ and $\rho u_{i}^{b} u_{j}^{b}$ respectively.

Note that although we subtract the ambient uniform flow $U$ from $u_{i}$, the source term on the right hand side of (2.4) still comprises of both linear and non-linear fluctuation terms. The linear terms are known to account for the jet mean flow refraction effects and should be most appropriately treated as a propagation effects rather than sources (Lighthill 1952; Lilley 1974; Goldstein 2003). We do not take the jet mean flow propagation effects into account. Hence, when modelling the sources on the right hand side of (2.4), only the non-linear fluctuation terms (the Favre average is used for velocities, see Goldstein (2003) for example) are used. However one can expect this to be acceptable both at low frequencies and for an observer at $90^{\circ}$ to the jet centreline, where the refraction effects are negligible. Now provided the source terms are known, (2.4) can be solved by making use of the Green's function satisfying appropriate boundary conditions. In the following sections, the Green's function is developed first and the source term is obtained by performing CFD calculations.

\subsubsection{The acoustic Green's function}

From (2.4), the Green's function satisfies the convective wave equation

$$
\left(\mathrm{i} \omega+U \frac{\partial}{\partial x_{1}}\right)^{2} G(\boldsymbol{x} ; \boldsymbol{y})-c_{0}^{2} \nabla^{2} G(\boldsymbol{x} ; \boldsymbol{y})=\delta(\boldsymbol{x}-\boldsymbol{y}) .
$$




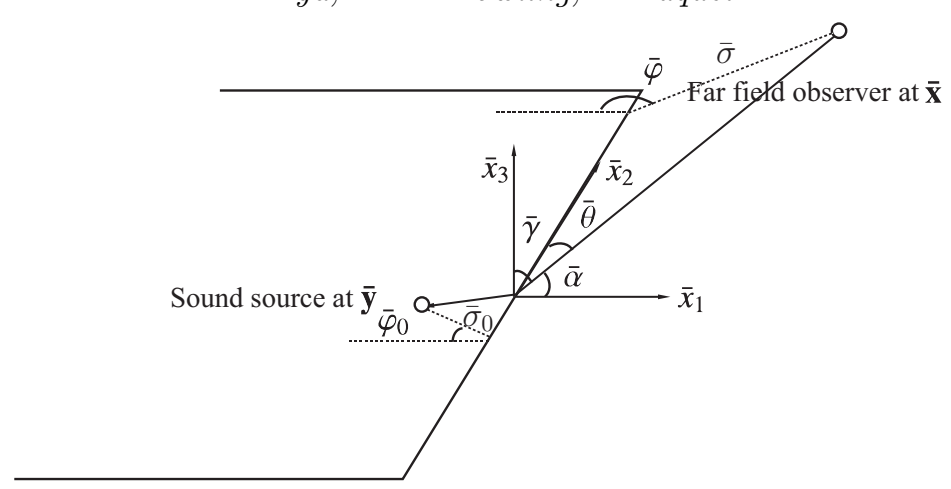

FiguRE 3. The schematic illustration of the stretched coordinates.

This equation, together the rigid-wall boundary conditions at the upper and lower surfaces of the flat plate, needs to be solved to obtain the Green's function.

Letting $k=\omega / c_{0}, M=U / c_{0}$, and $\beta^{2}=1-M^{2}$, and then making the coordinate transformation, $x_{1}=\bar{x}_{1}, x_{2}=\bar{x}_{2} / \beta, x_{3}=\bar{x}_{3} / \beta, y_{1}=\bar{y}_{1}, y_{2}=\bar{y}_{2} / \beta$ and $y_{3}=\bar{y}_{3} / \beta$, we can show that the solution to (2.5) (satisfying the rigid-wall boundary conditions on the upper and lower surfaces of the semi-infinite plate) can be found (Macdonald 1915; Lyu \& Dowling 2016) as

$$
G_{f}=\frac{\beta^{2} \mathrm{e}^{-\mathrm{i} \frac{k M}{\beta^{2}} \bar{y}_{1}}}{4 \pi c_{0}^{2}}\left(\frac{\mathrm{e}^{-\mathrm{i} \frac{k}{\beta^{2}} R}}{R} E\left(u_{R}\right)+\frac{\mathrm{e}^{-\mathrm{i} \frac{k}{\beta^{2}} R^{\prime}}}{R^{\prime}} E\left(u_{R^{\prime}}\right)\right),
$$

where $E(x)$ is an error function defined by

$$
E(x)=\frac{\mathrm{e}^{\mathrm{i} \pi / 4}}{\sqrt{\pi}} \int_{-\infty}^{x} \mathrm{e}^{-\mathrm{i} u^{2}} \mathrm{~d} u
$$

and $R$ and $R^{\prime}$ are given by

$$
\begin{aligned}
& R=\sqrt{\left(x_{1}-y_{1}\right)^{2}+\beta^{2}\left(x_{2}-y_{2}\right)^{2}+\beta^{2}\left(x_{3}-y_{3}\right)^{2}}, \\
& R^{\prime}=\sqrt{\left(x_{1}+y_{1}\right)^{2}+\beta^{2}\left(x_{2}+y_{2}\right)^{2}+\beta^{2}\left(x_{3}+y_{3}\right)^{2}} .
\end{aligned}
$$

Here

$$
\begin{aligned}
u_{R} & =2 \sqrt{\frac{k \bar{\sigma} \bar{\sigma}_{0}}{\beta^{2}(S+R)}} \cos \left(\frac{\bar{\varphi}-\bar{\varphi}_{0}}{2}\right), \\
u_{R^{\prime}} & =2 \sqrt{\frac{k \bar{\sigma} \bar{\sigma}_{0}}{\beta^{2}\left(S+R^{\prime}\right)}} \cos \left(\frac{\bar{\varphi}+\bar{\varphi}_{0}}{2}\right),
\end{aligned}
$$

where $S=\sqrt{\left(\bar{\sigma}+\bar{\sigma}_{0}\right)^{2}+\left(\bar{z}-\bar{z}_{0}\right)^{2}}$ and $(\bar{\sigma}, \bar{\varphi}, \bar{z})$ and $\left(\bar{\sigma}_{0}, \bar{\varphi}_{0}, \bar{z}_{0}\right)$ denote the corresponding cylindrical coordinates of the observer location and sound source location in the stretched Cartesian coordinate system $\left(\bar{x}_{1}, \bar{x}_{2}, \bar{x}_{3}\right)$ respectively, as shown in figure 3 . It is straightforward to show that

$$
\begin{array}{r}
\bar{\sigma}=\sqrt{x_{1}^{2}+\beta^{2} x_{3}^{2}}, \quad \bar{\sigma}_{0}=\sqrt{y_{1}^{2}+\beta^{2} y_{3}^{2}} \\
\cos (\bar{\varphi})=\frac{-x_{1}}{\bar{\sigma}}, \quad \cos \left(\bar{\varphi}_{0}\right)=\frac{-y_{1}}{\bar{\sigma}_{0}} \\
\bar{z}=\beta x_{2}, \quad \bar{z}_{0}=\beta y_{2}
\end{array}
$$


Similarly, the corresponding spherical coordinates of the observer and source positions in the stretched coordinate system are represented by $(\bar{r}, \bar{\theta}, \bar{\varphi})$ and $\left(\bar{r}_{0}, \bar{\theta}_{0}, \bar{\varphi}_{0}\right)$, respectively.

When the far-field observer assumption is invoked, the second derivatives of the Green's function can be obtained as

$$
\begin{aligned}
& \frac{\partial^{2} G(\boldsymbol{x} ; \boldsymbol{y})}{\partial y_{2}^{2}}=A\left(\boldsymbol{x}, y_{1}, y_{2}\right) k^{2} D_{22}^{0}\left(y_{1}, y_{3}\right), \\
& \frac{\partial^{2} G(\boldsymbol{x} ; \boldsymbol{y})}{\partial y_{1} \partial y_{2}}=A\left(\boldsymbol{x}, y_{1}, y_{2}\right) k^{2}\left(D_{12}^{0}\left(y_{1}, y_{3}\right)+\sqrt{\frac{1}{k \bar{\sigma}_{0}}} D_{12}^{1}\left(y_{1}, y_{3}\right)\right), \\
& \frac{\partial^{2} G(\boldsymbol{x} ; \boldsymbol{y})}{\partial y_{2} \partial y_{3}}=A\left(\boldsymbol{x}, y_{1}, y_{2}\right) k^{2}\left(D_{23}^{0}\left(y_{1}, y_{3}\right)+\sqrt{\frac{1}{k \bar{\sigma}_{0}}} D_{23}^{1}\left(y_{1}, y_{3}\right)\right), \\
& \frac{\partial^{2} G(\boldsymbol{x} ; \boldsymbol{y})}{\partial y_{1}^{2}}=A\left(\boldsymbol{x}, y_{1}, y_{2}\right) k^{2}\left(D_{11}^{0}\left(y_{1}, y_{3}\right)+\sqrt{\frac{1}{k \bar{\sigma}_{0}}} D_{11}^{1}\left(y_{1}, y_{3}\right)+\left(\sqrt{\frac{1}{k \bar{\sigma}_{0}}}\right)^{3} D_{11}^{2}\left(y_{1}, y_{3}\right)\right), \\
& \frac{\partial^{2} G(\boldsymbol{x} ; \boldsymbol{y})}{\partial y_{3}^{2}}=A\left(\boldsymbol{x}, y_{1}, y_{2}\right) k^{2}\left(D_{33}^{0}\left(y_{1}, y_{3}\right)+\sqrt{\frac{1}{k \bar{\sigma}_{0}}} D_{33}^{1}\left(y_{1}, y_{3}\right)+\left(\sqrt{\frac{1}{k \bar{\sigma}_{0}}}\right)^{3} D_{33}^{2}\left(y_{1}, y_{3}\right)\right), \\
& \frac{\partial^{2} G(\boldsymbol{x} ; \boldsymbol{y})}{\partial y_{1} \partial y_{3}}=A\left(\boldsymbol{x}, y_{1}, y_{2}\right) k^{2}\left(D_{13}^{0}\left(y_{1}, y_{3}\right)+\sqrt{\frac{1}{k \bar{\sigma}_{0}}} D_{13}^{1}\left(y_{1}, y_{3}\right)+\left(\sqrt{\frac{1}{k \bar{\sigma}_{0}}}\right)^{3} D_{13}^{2}\left(y_{1}, y_{3}\right)\right),
\end{aligned}
$$

where

$$
A\left(\boldsymbol{x}, y_{1}, y_{2}\right)=\frac{\beta^{2} \mathrm{e}^{-\mathrm{i} \frac{k}{\beta^{2}} \bar{r}} \mathrm{e}^{\mathrm{i} \frac{k M}{\beta^{2}} x_{1}}}{4 \pi c_{0}^{2} \bar{r}} \mathrm{e}^{\mathrm{i} \frac{k}{\beta^{2}}(-M+\cos \bar{\alpha}) y_{1}} \mathrm{e}^{\mathrm{i} \frac{k}{\beta} \cos \bar{\theta} y_{2}},
$$

and the detailed expressions for $D_{i j}^{k}$ are shown in the appendix. The terms $D_{i j}^{k}$ are properly bounded functions and determine the directivity patterns of the radiation from the corresponding quadrupoles. Therefore the noise enhancement arises from the terms $\sqrt{1 /\left(k \bar{\sigma}_{0}\right)}$ and $\sqrt{1 /\left(k \bar{\sigma}_{0}\right)^{3}}$ appearing in front of $D_{i j}^{1}$ and $D_{i j}^{2}$ respectively. When the frequency is sufficiently low, or the quadrupole source is sufficiently close to the edge of the flat plate, the far-field sound is dominated by the term involving $\sqrt{1 /\left(k \bar{\sigma}_{0}\right)}$ in the derivatives normal to the 2-axis, and is much larger than that when the plate is absent. The derivatives with one $y_{2}$ derivative have leading terms involving $\sqrt{1 /\left(k \bar{\sigma}_{0}\right)}$, and therefore are not as efficient as those derivatives involving $\sqrt{1 /\left(k \bar{\sigma}_{0}\right)^{3}}$ at low frequencies. Equation (2.10), however, does not involve enhanced terms, hence quadrupoles aligned with the 2-axis (corresponding to $\partial^{2} G(\boldsymbol{x} ; \boldsymbol{y}) / \partial y_{2}^{2}$ ) are the least efficient sound sources when close to the edge.

\subsubsection{CFD flow field calculation}

We have obtained the Green's function for the scattering problem in the preceding section. In order to solve (2.4) to determine the sound from the Lighthill quadrupoles, we need to obtain the source terms shown on the right hand side of (2.4). Unsteady numerical simulations such as Large Eddy Simulations (LES) are well suited for such a purpose. However, to perform an LES study is computationally expensive. The usual time required even on a massively parallel computer can vary from a few days to many weeks depending on practical needs. To avoid this difficulty attempts to use time-averaged flow calculations to predict isolated jet noise have been made by many authors. For example both Khavaran et al. (1994) and Bailly et al. (1994, 1996) used time-averaged calculations with turbulence statistics obtained from $k-\epsilon$ turbulence model for predicting supersonic jet noise. In the work of Bechara et al. (1995) a Reynolds-Averaged NavierStokes (RANS) calculation incorporating $k-\epsilon$ model for turbulence to characterise the 


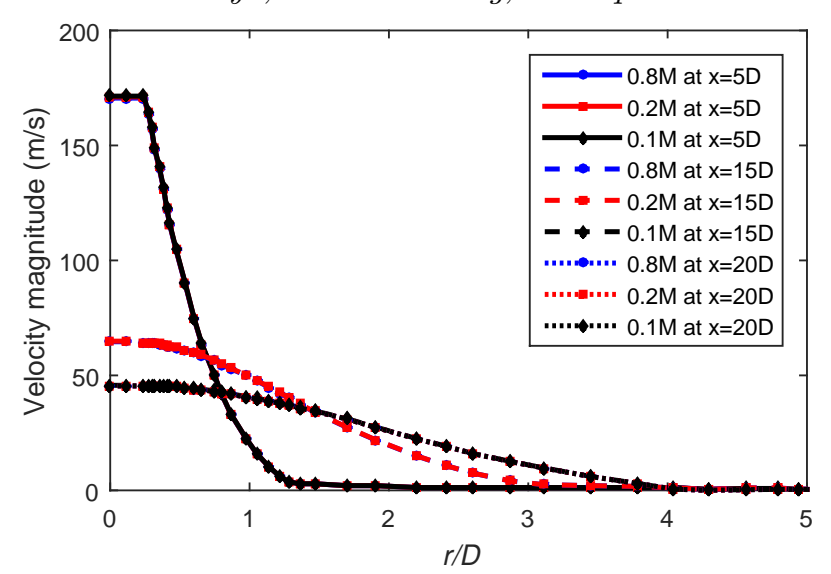

FiguRE 4. Velocity distributions in the radial direction using different meshes consisting of 0.8 million, 0.2 million and 0.1 million cells respectively.

sound sources was used to predict the noise of both simple and coaxial jets. Later Tam \& Auriault (1999) further explored this approach and successfully predicted jet noise at $90^{\circ}$ to the jet by proposing an empirical sound source model analogous to gas kinetic theory. Moreover, the three empirical constants in Tam's work were obtained by fitting to the far-field noise. On the contrary, the work by Karabasov et al. (2010) is based on Goldstein's acoustic analogy theory (Goldstein 2003) and the proportionality constants used in its source model are obtained from analysing LES data, which therefore contains little empiricism apart from that of the $k-\epsilon$ model for turbulence. The predicted noise at various observer angles to the jet are found to agree well with experiments. In this study we adopt the same approach in order to yield fast predictions. Therefore in this section we perform a RANS study with the standard $k-\epsilon$ turbulence model, and the source terms are subsequently modelled by making use of the time-averaged flow variables from the RANS, as will be described in detail in the next section.

The commercial software ANSYS Fluent 16.0 is used to perform a RANS calculation of an isolated round jet with the same temperature as the ambient air. When the wing and flap are sufficiently away from the jet, it can be expected that little change of the flow field occurs. Thus, as a starting point we first calculate the flow field for an axi-symmetric jet. The computational domain is $30 D$ and $5 D$ in the streamwise and radial directions respectively, where $D$ is the jet nozzle diameter. Calculations using much larger computational domains have shown little difference from those using the domain described above. The current domain size is therefore used to yield both a fast convergence and sufficiently accurate results.

The mesh used in the calculation is generated using ANSYS Meshing 16.0. A structured quadrilateral mesh is used for the entire domain, and the mesh is much denser near the mixing layer and close to the jet. Near the mixing layer, the grid size is around $0.025 \mathrm{D}$ in the axial direction and $0.02 D$ in the radial direction. The grid size inside the nozzle $(r<0.5 D)$ in the radial direction is nearly uniformly $0.025 D$. Near the downstream boundary the grid size is around $0.2 D$. The mesh consists of around 0.1 million cells. To check the mesh independence, the calculations using meshes consisting of 0.2 million and 0.8 million cells were carried out and the results yielded little difference, as shown in figure 4. It is shown that this number of cells ( 0.1 million) is sufficient to generate rapidly converging and sufficiently accurate results. A Wall function approach is used to resolve the boundary layer inside the nozzle. The chosen "standard wall function" in 
ANSYS Fluent is based on the work of Launder and Spalding (ANSYS 2015; Launder $\&$ Spalding 1974). The boundary layer mesh starts at around $y^{+}=50$, where $y^{+}$is the dimensionless distance of the first mesh layer to the wall based on the wall friction velocity (White 2005). The wall friction velocity $u^{*}$ is estimated using the log-law of the boundary layer (White 2005). The boundary layer is resolved by around 15 layers, which should be sufficient according to the Fluent Theory Guide (ANSYS 2015). Calculations using more layers show little difference for the mean and turbulent flow quantities.

The boundary conditions for boundaries both upstream and downstream are "pressure outlets" provided in ANSYS Fluent (ANSYS 2015). At the nozzle inlet boundary "pressure inlet" is used (ANSYS 2015), which specifies both the stagnation pressure and the stagnation temperature. The stagnation pressure and stagnation temperature are given on the inlet boundary such that a Mach number $M_{0}=0.5$ jet is obtained, where $M_{0}=U_{j} / c_{0}$ with $U_{j}$ being the mean jet exit velocity. The static temperature at the inlet boundary is the same as that of the ambient air, which is set to be $300 \mathrm{~K}$. The turbulence intensity at the "pressure inlet" is set to be $5 \%$, and studies using other values have shown little change to the calculated flow field, especially after the potential core.

The standard $k-\epsilon$ model for the turbulence is used, as it gives a more realistic core length (see figure 5 for example) compared to other turbulent models such as realizable $k-\epsilon$ model provided in ANSYS Fluent (ANSYS 2015). In fact although the potential core length varies, the difference that changing turbulent models causes on the turbulent flow quantities is not significant, especially for the locations $(x>4 D)$ where the sound generation is most efficient. Moreover, since the far-field sound depends on an integration over the whole turbulent flow field, little difference can be expected. As shown by Mohan et al. (2015), the use of many different turbulent models, such as $k-\epsilon, k-\omega$ etc, makes little difference to the far-field sound spectrum over the entire frequency range. Therefore we think that the use of $k-\epsilon$ model is sufficient for the current purpose. The compressible Reynolds-Averaged Navier-Stokes equations are solved, and the ideal-gas law for air is adopted while the ambient pressure is set to be $1 \mathrm{~atm}$. A model for the fourth-order space-time correlation function, as described in detail in the next section, can then be obtained from the flow variables $\bar{\rho}, \overline{\boldsymbol{u}}, k$ and $\epsilon$, which denote the mean density, mean velocities, turbulence intensity and turbulence dissipation rate respectively.

To validate the RANS simulation, the streamwise components of the mean and fluctuation velocities (root-mean-square value), both along the lip line and centre line of jet, are compared with the experimental data published by Bridges (Bridges \& Wernet 2010), as shown in figure 5. The details of Bridges' experiment will be discussed in details in section 2.2.1. From figure $5(\mathrm{a})$, it can be seen that the mean velocity profiles, in particular that on the lip line, agree well with experiment. On the centre line, the core length matches well with experimental data thought it appears that the velocity is slightly under predicted further downstream. Given that the Reynolds number for Bridge's experiment is twice that for the RANS simulation (as $D$ are 1 inch and 2 inches for the RANS and Bridge's experiment respectively) and the temperature ratio in the experiment is slightly different from that in the simulation, such agreement is good and the effect of any difference on the sound propagation can be expected to be negligible. Figure 5(b) shows the turbulent fluctuation velocity profiles on the jet lip line and centre lines. The root-mean-square value of $u_{1}^{\prime}$, where $u_{1}^{\prime}$ is the axial fluctuation velocity, is available in the experiment, but not from the RANS simulation. To facilitate comparison, we estimate this value from the turbulence intensity $k$ by assuming that the turbulence is isotropic. This assumption however is known to be not correct (Karabasov et al. 2010; Mohan et al. 2015), therefore we expect a slight under-prediction of the axial turbulent fluctuation velocities, which can be seen from figure 5(b). Apart from these uncertain- 


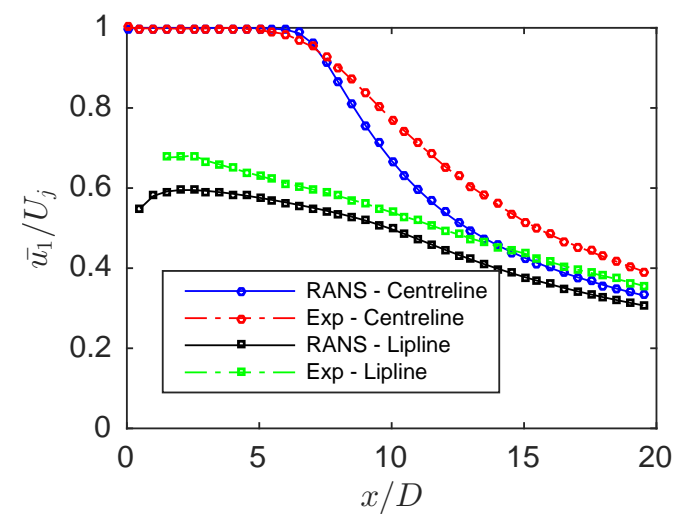

(a) Jet mean axial velocity

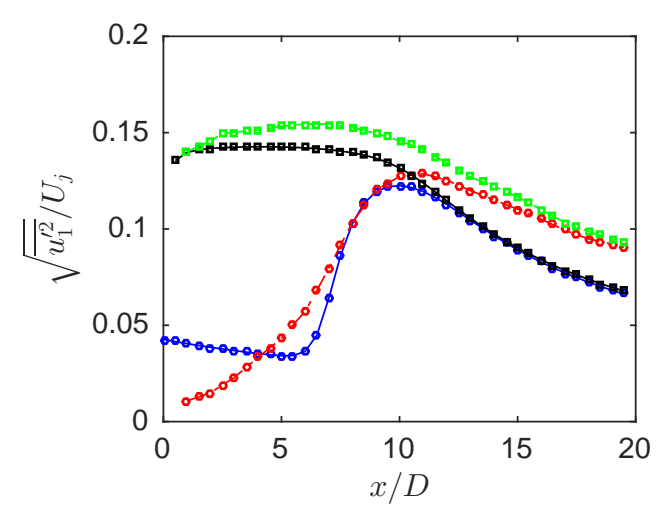

(b) Root-mean-square axial fluctuation velocity

Figure 5. Comparison of the jet mean and root-mean-square axial fluctuation velocities along the centre and lip lines between the RANS and experimental data. Isotropic turbulence is assumed for the RANS to obtain the root-mean-square axial fluctuation velocity from turbulence intensity $k$.

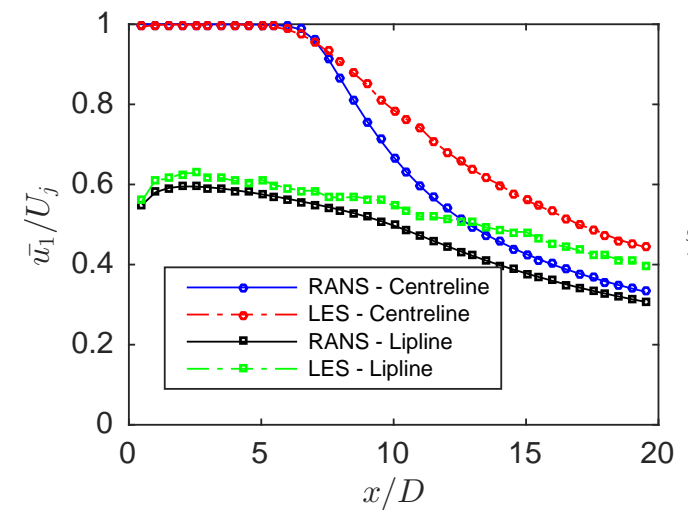

(a) Jet mean axial velocity

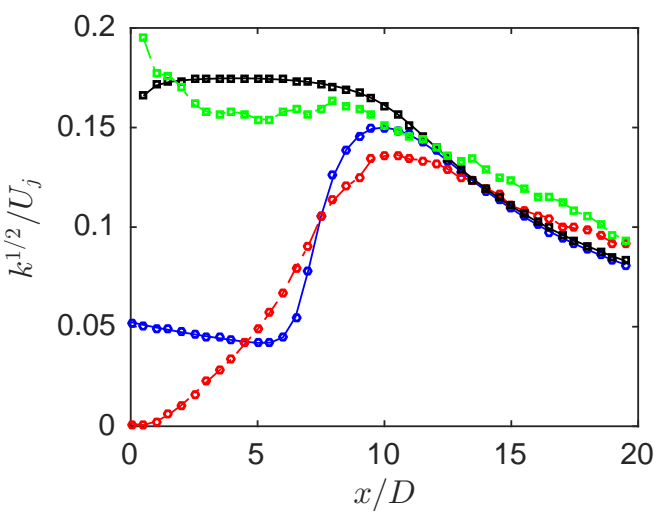

(b) Turbulence intensity

Figure 6. Comparison of the jet mean and turbulence intensity along the centre and lip lines between the RANS and LES results.

ties, it can be seem that an overall good agreement is achieved for data on the lip line. The results on the centre line also agrees well after about $x / D=6$. The over-prediction in the first few diameters is expected. This is because the inlet turbulence intensity is given a high-than-normal value of $5 \%$ so that the centre-line turbulence profiles near the downstream edge of the potential core can better agree with the experiment. However, as mentioned earlier, changing the inlet turbulence intensity has no effect on the flow field after the potential core.

As will be mentioned in the rest of this paper, an LES study is also performed with the same operating conditions as those in Bridges' experiment. Details of the LES can be found in section 2.2.1. It is therefore useful to compare the results of our RANS simulation to those obtained in the LES. Because we are now able to calculate the turbulence intensity directly from LES, we do not need to compare the root-mean-square of the axial fluctuation velocity by assuming isotropic turbulence for the RANS results. The results are shown in figure 6 . It can be seen that a similar good agreement to 
that shown in figure 5(a) for the mean axial velocities is achieved. However comparing figure $6(\mathrm{~b})$ to figure $5(\mathrm{~b})$, one sees a much better agreement for the turbulence intensity distribution, especially for the axial positions after the potential core. This shows that the cause for the under-prediction shown in figure 5(b) is indeed due to the assumption of isotropic turbulence (Karabasov et al. 2010; Mohan et al. 2015) and the RANS simulation is capable of predicting the jet flow accurately.

\subsubsection{Far-field sound due to Lighthill's quadrupoles}

Combining the acoustic Green's function, the model of the fourth-order space-time correlation function from Karabasov et al. (Karabasov et al. 2010) and the mean flow data from the RANS solution, we are now in a position to formulate the far-field sound power spectra. It is well established that the far-field sound perturbation can be written as

$$
\rho^{\prime}(\boldsymbol{x}, \omega)=\int_{V} \hat{T}_{i j}(\boldsymbol{y}, \omega) \frac{\partial^{2} G(\boldsymbol{x} ; \boldsymbol{y}, \omega)}{\partial y_{i} \partial y_{j}} \mathrm{~d}^{3} y .
$$

The integral in (2.17) is over the entire volume where $\hat{T}_{i j}(\boldsymbol{y}, \omega)$ is not second-order small. By making use of the linearized relationship $p^{\prime}(\boldsymbol{x}, \omega)=c_{0}^{2} \rho^{\prime}(\boldsymbol{x}, \omega)$ and $\Phi_{Q}(\boldsymbol{x}, \omega)=$ $\lim _{T \rightarrow \infty} \frac{\pi}{T} \overline{p^{\prime}(\boldsymbol{x}, \omega) p^{* *}(\boldsymbol{x}, \omega)}$, where $\Phi_{Q}(\boldsymbol{x}, \omega)$ represents the power spectral density (PSD) of far-field sound and $*$ denotes taking complex conjugate, it can be shown that

$$
\Phi_{Q}(\boldsymbol{x}, \omega)=c_{0}^{4} \int_{V_{y}} \int_{V_{\Delta y}} R_{i j k l}(\boldsymbol{y}, \Delta \boldsymbol{y}, \omega) I_{i j k l}(\boldsymbol{x}, \boldsymbol{y}, \Delta \boldsymbol{y}, \omega) \mathrm{d}^{3} \Delta y \mathrm{~d}^{3} y
$$

where

$$
\begin{aligned}
R_{i j k l}(\boldsymbol{y}, \Delta \boldsymbol{y}, \omega) & =\frac{1}{2 \pi} \int_{-\infty}^{\infty} \overline{T_{i j}(\boldsymbol{y}, t) T_{k l}(\boldsymbol{y}+\Delta \boldsymbol{y}, t+\tau)} \mathrm{e}^{-\mathrm{i} \omega \tau} \mathrm{d} \tau, \\
I_{i j k l}(\boldsymbol{x}, \boldsymbol{y}, \Delta \boldsymbol{y}, \omega) & =\frac{\partial^{2} G(\boldsymbol{x} ; \boldsymbol{y}, \omega)}{\partial y_{i} \partial y_{j}} \frac{\partial^{2} G^{*}(\boldsymbol{x} ; \boldsymbol{y}+\Delta \boldsymbol{y}, \omega)}{\partial y_{k} \partial y_{l}} .
\end{aligned}
$$

It has been known that the fourth-order space-time correlation function $R_{i j k l}(\boldsymbol{y}, \Delta \boldsymbol{y}, \tau)$ can be well represented by a Gaussian function as (Karabasov et al. 2010)

$$
\begin{aligned}
R_{i j k l}(\boldsymbol{y}, \Delta \boldsymbol{y}, \tau)= & A_{i j k l}(\boldsymbol{y}) \exp \left[-\frac{\left|\Delta y_{1}\right|}{\bar{u}_{1}(\boldsymbol{y}) \tau_{s}(\boldsymbol{y})}\right. \\
& \left.-\ln 2\left(\left(\frac{\Delta y_{1}-\bar{u}_{1}(\boldsymbol{y}) \tau}{l_{1}(\boldsymbol{y})}\right)^{2}+\left(\frac{\Delta y_{2}}{l_{2}(\boldsymbol{y})}\right)^{2}+\left(\frac{\Delta y_{3}}{l_{3}(\boldsymbol{y})}\right)^{2}\right)\right],
\end{aligned}
$$

where $A_{i j k l}(\boldsymbol{y})=C_{i j k l}(2 \bar{\rho} k)^{2}, l_{i}=c_{i} k^{3 / 2} / \epsilon$ and $\tau_{s}=c_{\tau} k / \epsilon$. The constants $C_{i j k l}, c_{i}$ and $c_{\tau}$ can be obtained by best fitting (2.20) to the space-time correlation data obtained from LES simulations. By analyzing the LES data (see more from Karabasov et al. (2010)), we found that $c_{1}, c_{2}, c_{3}, c_{\tau}$ are around $0.4,0.23,0.23,0.3$ respectively, which are close to those obtained by Karabasov et al. (2010), but also account for the anisotropy of the turbulence length scales (see Mohan et al. (2015)). $C_{i j k l}$ remain the same as those found by Karabasov et al. (2010). The fourth-order space-time correlation of the non-linear source term is known to be well modelled by (2.20) (see more details from Goldstein (2003) and Karabasov et al. (2010)). Using (2.20), the cross-spectra is thus obtained by 
performing the standard Fourier transformation, which yields

$$
\begin{aligned}
R_{i j k l}(\boldsymbol{y}, \Delta \boldsymbol{y}, \omega)= & \frac{l_{1}(\boldsymbol{y})}{2 \bar{u}_{1}(\boldsymbol{y}) \sqrt{\pi \ln 2}} A_{i j k l}(\boldsymbol{y}) \exp \left[-\frac{l_{1}(\boldsymbol{y})^{2} \omega^{2}}{4 \bar{u}_{1}^{2}(\boldsymbol{y}) \ln 2}\right] \\
& \exp \left[-\frac{\left|\Delta y_{1}\right|}{\bar{u}_{1}(\boldsymbol{y}) \tau_{s}(\boldsymbol{y})}-\mathrm{i} \frac{\omega}{\bar{u}_{1}(\boldsymbol{y})} \Delta y_{1}-\ln 2\left(\left(\frac{\Delta y_{2}}{l_{2}(\boldsymbol{y})}\right)^{2}+\left(\frac{\Delta y_{3}}{l_{3}(\boldsymbol{y})}\right)^{2}\right)\right]
\end{aligned}
$$

The tensor $I_{i j k l}(\boldsymbol{x}, \boldsymbol{y}, \Delta \boldsymbol{y}, \omega)$ in (2.19) depends solely on the Green's function. Consequently, substituting the free-space Green's function or the one developed in the first part of this section into (2.18) yields results for an isolated jet or installed jet respectively.

\subsection{Near-field scattering}

Outside the jet mixing layer, there is a region of near-field pressure fluctuations, which is primarily induced by hydrodynamic instability waves and decays exponentially in the radial direction (Jordan \& Colonius 2013). In the frequency regime $S t>0.1$ the waves convect at a virtually constant speed $U_{c} \approx 0.6 \sim 0.8 U_{j}$ where $S t$ is the Strouhal number based on the jet diameter (Arndt et al. 1997; Tinney \& Jordan 2008; Gudmundsson \& Colonius 2011; Jordan \& Colonius 2013). Since the convection velocity is lower than the speed of sound, the pressure due to the field of a hydrodynamic wave decays exponentially in the radial direction (see the details in the following section) and therefore only contributes weakly to the far-field of an isolated jet. When acoustic analogy theories are adopted for the isolated jet, these evanescent waves are not regarded as quadrupole sources, since their magnitude of fluctuation is small enough to be regarded as linear (pseudo sound, thus their source strength would be second-order small). However, when a surface with sharp edges is present in the near-field of the jet, the previously nonradiating pressure field can be efficiently scattered into sound by the edge. Thus, this suggests that the Lighthill's quadrupole sources are not sufficient to correctly predict the far-field of the installed jet, and the sound scattered by the sharp edges of the aerofoil must be accounted for. In this section, the far-field sound due to the interaction between the near-field evanescent waves and the plate edge is modelled using Amiet's approach.

It is worth noting that the trailing-edge scattering mechanism of the hydrodynamic field has been suggested in several earlier works, for example those of Lawrence et al. (2011) and Bychkov \& Faranosov (2014). However, the term of hydrodynamic field is not always same as the evanescent wave mentioned here, for both non-linear and linear regions of hydrodynamic field exist. The evanescent wave mentioned here accounts for only the exponentially-decaying linear part (excluding both the non-linear and linear acoustic parts), the mechanisms of which cannot be captured by Lighthill's acoustic analogy. It should also be noted that the most heavily researched and cited convection velocity, which is around $0.6 \sim 0.8 U_{j}$, is primarily for the instability waves at frequencies around $S t=$ 0.3 . But whether this convection speed is still constant at very low frequencies is not yet known. In fact the constant-convection-velocity assumption has been called into question by several authors (Kerhervé et al. 2006). By analysing LES data we confirmed the frequency-dependence of the convection velocity and found that this convection velocity $U_{c}$ is significantly lower than $0.6 U_{j}$ at low frequencies, e.g. for $S t<0.1$. This frequency dependence is included in our model.

\subsubsection{The near-field evanescent waves}

As the near-field evanescent waves are scattered into sound nearby the trailing edge, it is useful to investigate its properties before we move on to model the scattering mechanis$\mathrm{m}$, in particular its spatial correlation. To illustrate the idea, we consider here an isolated 


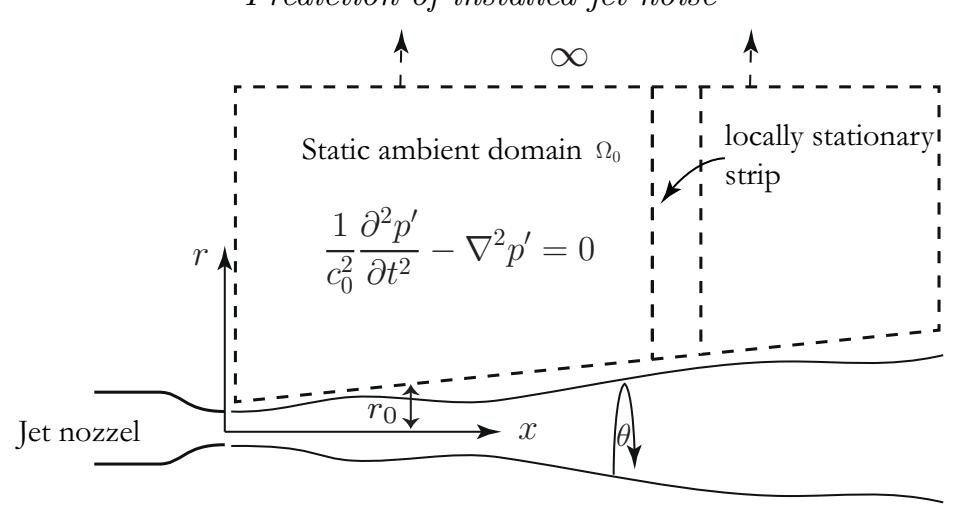

FiguRE 7. Schematic of the jet with a static ambient flow. $r, \theta$ and $x$ denotes the radial, azimuthal and streamwise coordinates respectively. Immediately outside the jet plume where the flow velocity virtually vanishes, the linearised Navier-Stokes equation resulted in the classical wave equation. Due to the spreading of the jet flow, only the pressure fluctuation in the narrow strip might be regarded as a stationary function of $x$.

jet with static ambient flow, as shown in figure 7. Note that we work with an isolated jet in this section, thus it is sensible to temporarily switch to a cylindrical coordinate system with its origin located at the centre of the nozzle exit, as shown in figure 7 . The axial and radial coordinates are denoted by $x$ and $r$ respectively. At any axial positions $x$, the mean jet velocity decays quickly (outside the potential core) as $r$ increases. Therefore, the velocity effectively vanishes when the radial distance from the jet centre line is larger than a value $r_{0}$. Due to the spreading effect of the jet and the self-similarity exhibited by the velocity distribution along the radial lines, $r_{0}$ would increase as $x$ increases. Consequently, the pressure fluctuation when $r>r_{0}$, as shown by the domain $\Omega_{0}$ in figure 7 , is governed by the classical wave equation resulting from the linearisation of the perturbed Navier-Stokes equations, which is standard and not repeated here.

The solution of the reduced wave equation for frequency $\omega$ in $\Omega_{0}$ is also standard. If we are only interested the radially-decaying components (excluding the oscillating acoustic components), the solution must be of the form

$$
p^{\prime}(\omega, \boldsymbol{x})=\sum_{m=-\infty}^{\infty} \int_{-\infty}^{\infty} \hat{p}\left(\omega, m, k_{x}\right) K_{m}\left(\sqrt{k_{x}^{2}-k^{2}} r\right) \mathrm{e}^{\mathrm{i} m \theta} \mathrm{e}^{-\mathrm{i} k_{x} x} \mathrm{~d} k_{x},
$$

where $\theta$ is the azimuthal angle, $K_{m}(r)$ is the $m$-th modified Bessel function of the second kind and we have made the use of the fact that the exponentially growing solution $I_{m}(r)$ tending to infinity as $r \rightarrow \infty$ must be excluded. As we have excluded the solutions corresponding to acoustic fluctuations, the integral interval of $k_{x}$ should strictly be from $-\infty$ to $-k$ and from $k$ to $\infty$. However since this does not affect our following derivation, we will use the interval from $-\infty$ to $\infty$ for simplicity.

Since the flow field is turbulent in time $t, \hat{p}\left(\omega, m, k_{x}\right)$ would be a statistical quantity with respect to $\omega$. In addition, the turbulence flow also results in the randomness of the boundary condition of the domain $\Omega_{0}$, it can be expected that $\hat{p}\left(\omega, m, k_{x}\right)$ would be a statistical quantity with respect to $m$ and $k_{x}$ as well. Therefore the cross power spectral density of two points located at the same $x$ and $\theta$ but at $r_{1}$ and $r_{2}$ respectively would be 


$$
\begin{aligned}
R\left(\omega ; r_{1}, r_{2}\right) \equiv & \lim _{T \rightarrow \infty} \frac{\pi}{T} \overline{\hat{p}\left(\omega, r_{1}\right) \hat{p}^{*}\left(\omega, r_{2}\right)} \\
= & \lim _{T \rightarrow \infty} \frac{\pi}{T} \sum_{m=-\infty}^{\infty} \sum_{n=-\infty}^{\infty} \int_{-\infty}^{\infty} \int_{-\infty}^{\infty} \frac{\hat{p}\left(\omega, m, k_{x}\right) \hat{p}^{*}\left(\omega, n, k_{x}^{\prime}\right)}{} \\
& \times K_{m}\left(\sqrt{k_{x}^{2}-k^{2}} r_{1}\right) K_{n}\left(\sqrt{k_{x}^{\prime 2}-k^{2}} r_{2}\right) \mathrm{e}^{\mathrm{i}(m-n) \theta} \mathrm{e}^{-\mathrm{i}\left(k_{x}-k_{x}^{\prime}\right) x} \mathrm{~d} k_{x} \mathrm{~d} k_{x}^{\prime}
\end{aligned}
$$

where, $R\left(\omega ; r_{1}, r_{2}\right)$ denotes the cross power spectral density of the aforementioned two points and $2 T$ is the time interval for performing temporal Fourier transform $p(\omega, x)$. The overbar and star denote ensemble average and complex conjugate respectively.

Let us assume that $p^{\prime}(\omega, \boldsymbol{x})$ is a statistically-stationary function of $x$ and $\theta$ (Tinney \& Jordan 2008), which implies

$$
\lim _{T \rightarrow \infty} \frac{\pi}{T} \overline{\hat{p}\left(\omega, m, k_{x}\right) \hat{p}^{*}\left(\omega, n, k_{x}^{\prime}\right)}=P\left(\omega, m, k_{x}\right) \delta\left(k_{x}-k_{x}^{\prime}\right) \delta_{n m},
$$

where $\delta(x)$ and $\delta_{m n}$ are the conventional generalized $\delta$ function and Kronecker delta respectively. Substituting (2.24) into (2.23) yields

$$
R\left(\omega ; r_{1}, r_{2}\right)=\sum_{m=-\infty}^{\infty} \int_{-\infty}^{\infty} P\left(\omega, m, k_{x}\right) K_{m}\left(\sqrt{k_{x}^{2}-k^{2}} r_{1}\right) K_{m}\left(\sqrt{k_{x}^{2}-k^{2}} r_{2}\right) \mathrm{d} k_{x} .
$$

If we define the spectral correlation coefficient as

$$
\eta\left(\omega ; r_{1}, r_{2}\right) \equiv \frac{\left|\overline{\hat{p}\left(\omega, r_{1}\right) \hat{p}^{*}\left(\omega, r_{2}\right)}\right|}{\sqrt{\mid \hat{\left|\hat{p}\left(\omega, r_{1}\right)\right|^{2}} \overline{\left|\hat{p}\left(\omega, r_{2}\right)\right|^{2}}}},
$$

it follows from $(2.23)$ and $(2.25)$ that

$$
\begin{aligned}
& \eta\left(\omega ; r_{1}, r_{2}\right)=\frac{\left|R\left(\omega ; r_{1}, r_{2}\right)\right|}{\sqrt{R\left(\omega ; r_{1}, r_{1}\right) R\left(\omega ; r_{2}, r_{2}\right)}} \\
& =\frac{\left|\sum_{m=-\infty}^{\infty} \int_{-\infty}^{\infty} P\left(\omega, m, k_{x}\right) K_{m}\left(\gamma r_{1}\right) K_{m}\left(\gamma r_{2}\right) \mathrm{d} k_{x}\right|}{\sqrt{\sum_{m=-\infty}^{\infty} \int_{-\infty}^{\infty} P\left(\omega, m, k_{x}\right)\left[K_{m}\left(\gamma r_{1}\right)\right]^{2} \mathrm{~d} k_{x} \sum_{m=-\infty}^{\infty} \int_{-\infty}^{\infty} P\left(\omega, m, k_{x}\right)\left[K_{m}\left(\gamma r_{2}\right)\right]^{2} \mathrm{~d} k_{x}}},
\end{aligned}
$$

where $\gamma \equiv \sqrt{k_{x}^{2}-k^{2}}$ denotes the radial decay of the evanescent waves. Although using Cauchy-Schwartz inequality equation it can be readily shown that $\eta\left(\omega, r_{1}, r_{2}\right) \leqslant 1$, it is obvious that the summation over circumferential mode $m$ makes it hard to reach any useful conclusion about how much correlation there exists between the two points. Therefore, we try to remove the summation through a modal decomposition in the $\theta$ direction of the near-field pressure on two co-axial circles at the same $x$.

The mathematical derivation is similar, except that we write

$$
p^{\prime}(\omega, m, r, x)=\int_{-\infty}^{\infty} \hat{p}\left(\omega, m, k_{x}\right) K_{m}\left(\sqrt{k_{x}^{2}-k^{2}} r\right) \mathrm{e}^{-\mathrm{i} k_{x} x} \mathrm{~d} k_{x} .
$$

Repeating the above steps yields

$$
R\left(\omega, m ; r_{1}, r_{2}\right)=\int_{-\infty}^{\infty} P\left(\omega, m, k_{x}\right) K_{m}\left(\sqrt{k_{x}^{2}-k^{2}} r_{1}\right) K_{m}\left(\sqrt{k_{x}^{2}-k^{2}} r_{2}\right) \mathrm{d} k_{x}
$$


If we define the modal spectral correlation coefficient as

$$
\eta\left(\omega, m ; r_{1}, r_{2}\right) \equiv \frac{\left|\overline{\hat{p}\left(\omega, m, r_{1}\right) \hat{p}^{*}\left(\omega, m, r_{2}\right)}\right|}{\sqrt{\overline{\left|\hat{p}\left(\omega, m, r_{1}\right)\right|^{2}}\left|\hat{p}\left(\omega, m, r_{2}\right)\right|^{2}}},
$$

it follows that

$$
\begin{aligned}
& \eta\left(\omega, m ; r_{1}, r_{2}\right)=\frac{\left|R\left(\omega, m ; r_{1}, r_{2}\right)\right|}{\sqrt{R\left(\omega, m ; r_{1}, r_{1}\right) R\left(\omega, m ; r_{2}, r_{2}\right)}} \\
& =\frac{\left|\int_{-\infty}^{\infty} P\left(\omega, m, k_{x}\right) K_{m}\left(\sqrt{k_{x}^{2}-k^{2}} r_{1}\right) K_{m}\left(\sqrt{k_{x}^{2}-k^{2}} r_{2}\right) \mathrm{d} k_{x}\right|}{\sqrt{\int_{-\infty}^{\infty} P\left(\omega, m, k_{x}\right)\left[K_{m}\left(\sqrt{k_{x}^{2}-k^{2}} r_{1}\right)\right]^{2} \mathrm{~d} k_{x} \int_{-\infty}^{\infty} P\left(\omega, m, k_{x}\right)\left[K_{m}\left(\sqrt{k_{x}^{2}-k^{2}} r_{2}\right)\right]^{2} \mathrm{~d} k_{x}}} .
\end{aligned}
$$

It is clear that for a fixed frequency $\omega$ and circumferential mode number $m$ the coefficient would depend on the specific form of function $P\left(\omega, m, k_{x}\right)$. If for example, the local convective speed of the evanescent wave is a roughly-constant value, $U_{c}(\omega, m)$, which would mean $P\left(\omega, m, k_{x}\right)$ obtains a large value around $\bar{k}_{x}=\omega / U_{c}$ (note $\bar{k}_{x}$ is a function of both $\omega$ and $m$ ), then it follows that the approximation

$$
P\left(\omega, m, k_{x}\right)=P(\omega, m) \delta\left(k_{x}-\bar{k}_{x}\right)
$$

holds. Equation (2.31) can thus simplify to

$$
\eta\left(\omega, m ; r_{1}, r_{2}\right)=\frac{\left|P(\omega, m) K_{m}\left(\sqrt{\bar{k}_{x}^{2}-k^{2}} r_{1}\right) K_{m}\left(\sqrt{\bar{k}_{x}^{2}-k^{2}} r_{2}\right)\right|}{\sqrt{P(\omega, m)\left[K_{m}\left(\sqrt{k_{x}^{2}-k^{2}} r_{1}\right)\right]^{2} P(\omega, m)\left[K_{m}\left(\sqrt{k_{x}^{2}-k^{2}} r_{2}\right)\right]^{2}}}=1 .
$$

In another words, if the convective velocity of the evanescent waves were indeed dominated by a fixed value for each $m$ and $\omega$, then we would obtain a perfect correlation between the modal spectra of the pressure on two co-axial circles at the same $x$. Therefore the value of $\eta\left(\omega, m ; r_{1}, r_{2}\right)$ can be used to determine whether there exists such a dominant convection velocity for each mode number $m$ and frequency $\omega$.

However it should be noted that in order to reach this conclusion, we made use of the assumption that $p^{\prime}(\omega, \boldsymbol{x})$ is a statistically-stationary function of $x$. However we know that the near-field instability waves also grow and decay slowly. To avoid this difficulty we only need to consider the pressure inside a sufficiently narrow strip shown in figure 7 . As the growth and decay occur slowly, we expect the locally stationary assumption to be a good approximation within this strip. All the preceding derivation and conclusion remain unchanged, except quantities are interpreted in a local sense and will depend on $x$. For example $\bar{k}_{x}$ is now also a function of $x$, which implies that the convection velocity can also vary as $x$ changes.

To examine to what extent the dominant-convection-velocity assumption serves as a good approximation, we carried out a hybrid RANS-implicit LES study for an isolated single stream jet from the Bridges' experimental data (set point 3) (Bridges \& Wernet 2010). The jet diameter $D=5.08 \mathrm{~cm}$, jet Mach number $M_{0}=0.5$, jet temperature ratio $T_{j} / T_{\infty}=0.95$ and the nozzle pressure ratio (ratio of plenum pressure to atmospheric pressure) NPR $=1.197$. The jet Reynolds number is $5.8 \times 10^{5}$. The LES solver discretized Favre-averaged compressible Navier-Stokes equations with an unstructured non-orthogonal, second order finite volume scheme. The fluxes are approximated with the Roe method, where the central part is given by a Kinetic energy preserving scheme (Jameson 2008) with a fourth order dissipative term. In the active LES flow re- 


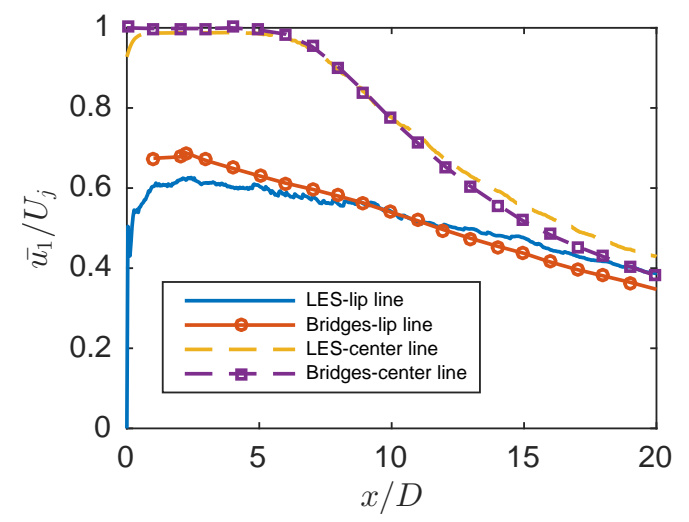

(a) Jet mean axial velocity

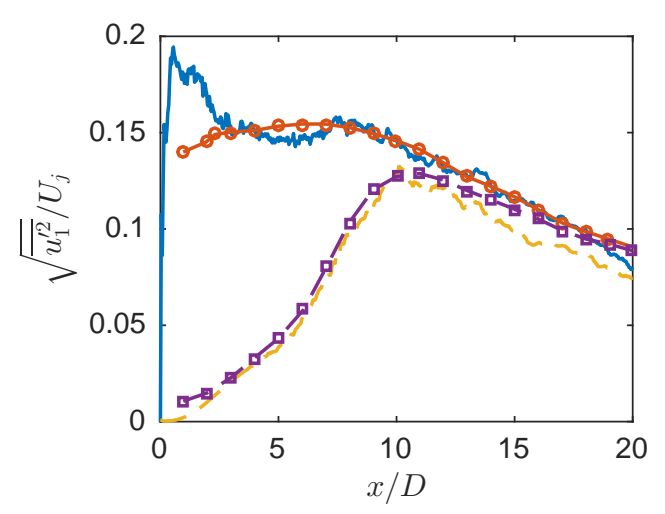

(b) Root-mean-square axial fluctuation velocity

Figure 8. Comparison of the jet mean and turbulent fluctuation velocity along centre line and lip line between the LES and experimental data.

gion of interest the dissipation is kept to a minimum value, which gives stable solution. Outside the LES region dissipation is increased for the sponge region to suppress the reflecting waves from the boundaries. Implicit LES relies on this numerical dissipation to remove sub-grid scales without any sub-grid model. A dual-time step method is used to advance the solution implicitly in time. The turbulent flow is developed in a pipe of length $4 D$ before exiting the nozzle. A RANS layer, with Spalart-Allmaras turbulence model, is applied in the near wall region of the pipe, which helped to reduce the grid resolution requirements. The computational domain is stretched in the streamwise $(-20 D \sim 100 D)$ and radial $(60 D)$ directions, with, as mentioned above, sponge zones at the boundaries. A structured axi-symmetric mesh of 24 million cells is used. The mesh being used can yield a satisfactory frequency resolution up to $S t=2$. The details of numerical methodology and grid independent studies have been discussed for various single stream jets by Naqavi et al. (2016) and the current grid distribution is the same as the validation case. To validate the LES simulation, we compared the calculated solution with Bridges experimental data (Bridges \& Wernet 2010). Both the mean flow and the turbulent fluctuation velocity profiles along the jet center line and lip line are compared. The results are shown in figures $8(\mathrm{a})$ to $8(\mathrm{~b})$. It can be seen that excellent agreement between the experimental and LES data is achieved for $x / D>2$. The disagreement for $x / D<2$ is expected because the flow velocity is very sensitive to the inlet boundary conditions and the inlet boundary conditions in experiments are very difficult to replicate.

Figure 9 shows the modal spectral correlation coefficient between pressure fluctuations on a pair of coaxial circles at different radial positions using the LES data. The axial position is $x / D=6$ and the correlation coefficient is defined between the circle at $r=$ $1.8 D$ and other circles at $r=1.9 D, 2.0 D, 2.1 D, 2.5 D, 3.0 D$ respectively. Only modes $m=0$ and $m= \pm 1$ are shown as the energy contained in mode $m$ reduces quickly as $m$ increases. This has been observed in several experimental studies, for example the experimental work of Tinney et al. (2008a) and Tinney \& Jordan (2008). From figure 9 it appears that the coefficient is close to 1 at low frequencies for either mode 0 or $( \pm) 1$. This implies that the assumption of a dominant convection velocity is valid. The strong oscillation at high frequencies $S t>0.2$ might well be due to acoustic contamination, particularly at large radial positions. However, as can be seen in the rest of this paper, the near-field pressure is only significant for frequencies satisfying $S t<0.2$. Therefore it 


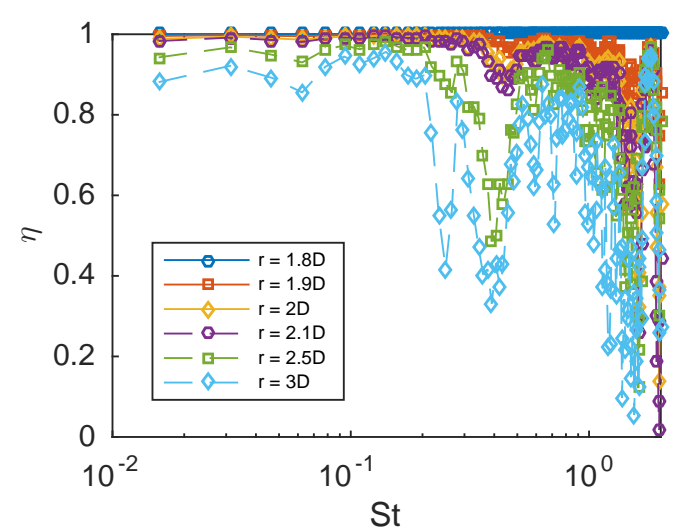

(a) Mode 0

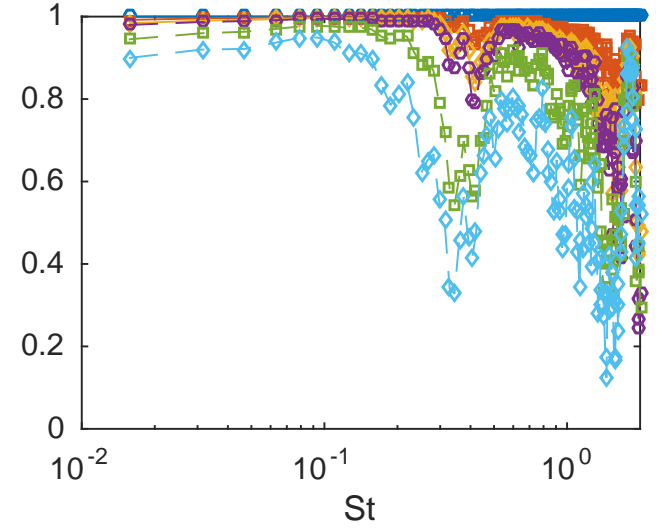

(b) Mode \pm 1

Figure 9. The modal spectral correlation coefficient $\eta$ between the point at $r=1.8 D$ and other points at different radial positions, all the points are at $x=6 D$.

is reasonable to assign a fixed $U_{c}$ for each $m$ and $\omega$ for the near-field evanescent waves in the scattering model to be developed in the next subsection.

To determine these convection velocities, we consider the modal PSD of the near-field pressure fluctuation along the circle located at the axial position $x$ and radial position $r$. Letting the correlation-defined spectrum

$$
\Pi(\omega, m ; r)=\lim _{T \rightarrow \infty} \frac{\pi}{T}\left|\frac{\overline{\hat{p}\left(\omega, m, r_{r e f}\right) \hat{p}^{*}(\omega, m, r)}}{\sqrt{\mid \hat{\left.\hat{p}\left(\omega, m, r_{r e f}\right)\right|^{2}}}}\right|^{2},
$$

where $r_{r e f}$ denotes the $r$ at a fixed reference point, then using (2.32), we have

$$
\Pi(\omega, m ; r)=P(\omega, m) K_{m}^{2}\left(\sqrt{\bar{k}_{x}^{2}-k^{2}} r\right) .
$$

Hence the reduced power spectrum

$$
\bar{\Pi}(\omega, m ; r)=\frac{\Pi(\omega, m ; r)}{K_{m}^{2}\left(\sqrt{\bar{k}_{x}^{2}-k^{2}} r\right)}=P(\omega, m)
$$

would be independent of $r$ and the reduced power spectra for any $r$ should collapse. $U_{c}$ can be evaluated from the collapsed radial decay rate $\gamma$.

Figure 10 shows the reduced power spectra for different $r$. An excellent data collapse is achieved for both mode 0 and mode $( \pm) 1$. This is another strong piece of evidence that there does indeed exist a dominant local convection velocity for the near-field evanescent waves for each $m$ and $\omega$. The convection velocities for mode $0,( \pm) 1$ and $( \pm) 2$ obtained in this way are shown in figure 11. As shown in figures 9 and 10 , both the high spatial correlation and successful collapse of the reduced spectra are only valid for frequencies $S t<0.2$. Therefore, the convection velocities are only shown in this lowfrequency regime. It can be seen that the convection velocity is much lower than $0.6 U_{j}$, which is heavily studied for instability waves at around $S t=0.3$. It is interesting to note that the frequency-dependent convection velocity curve obtained here is consistent with the finding of Kerhervé et al. (2006). From figure 11 we can find that the convection velocities for different azimuthal modes $m$ do not differ significantly from each other, especially for modes $0,( \pm) 1$ and $( \pm) 2$. This is consistent to the findings of Tinney et al. 


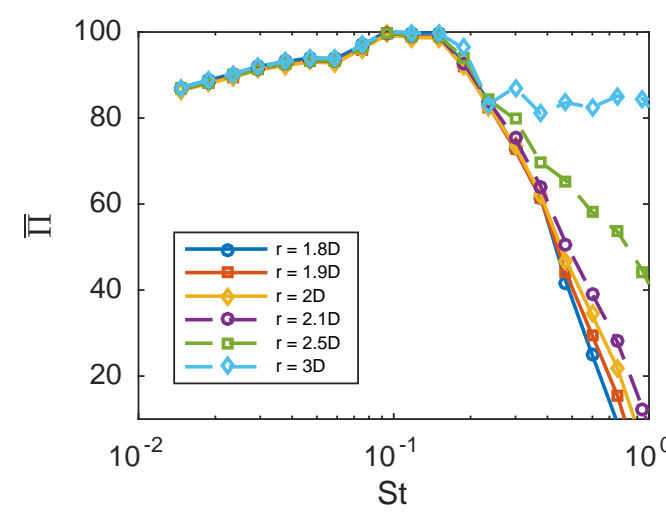

(a) Mode 0

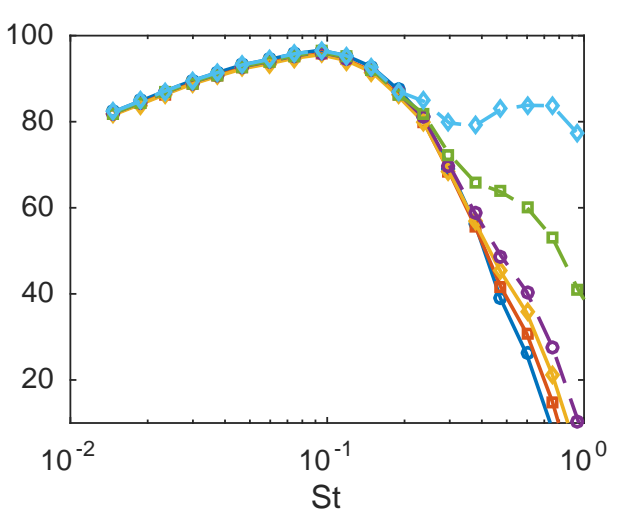

(b) Mode \pm 1

FigURE 10. The reduced power spectra of the near-field pressure fluctuation for points at $x=6 D$ and different radial positions.

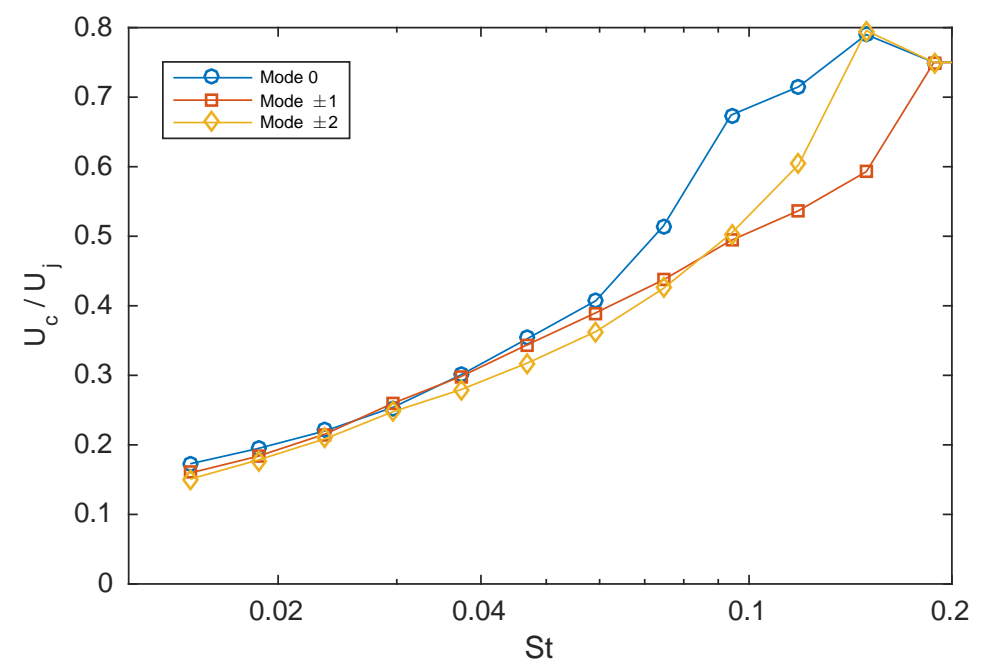

FiguRE 11. The convection velocities at different frequencies for different mode $m$ obtained by collapsing spectra at different radial positions but at the same $x / D=6$. Due to the dominant presence of acoustic waves at high frequencies, only low-frequency regime is shown.

(2008b) obtained using the POD technique by analysing the pressure-velocity coupling. This fact is used in the following sections to simplify the scattering model.

The reason of losing the spatial correlation for the near-field pressure, and hence the failure of collapsing modal PSD, for $S t>0.2$, is mostly due to the fact that the acoustic fluctuation is dominant in this frequency regime. To show this, we take the temporal and spatial Fourier transformations of the pressure along the straight line $r=3 D, \theta=0$ and show the results in figure 12. From figure 12(a) it can be clearly seen that the wavenumber spectrum of the near-field pressure at high frequencies falls entirely inside the acoustic cone. This means that the pressure is effectively acoustic fluctuations rather than due to evanescent waves. Note that from figure 12(a) we have further confirmed that the convection velocity is a frequency dependent quantity rather than a fixed constant between $0.6 U_{j}$ and $0.8 U_{j}$. To better demonstrate this the central region of figure $12(\mathrm{a})$ is expanded in figure 12(b). It is clear that at low frequencies the convection velocity is 


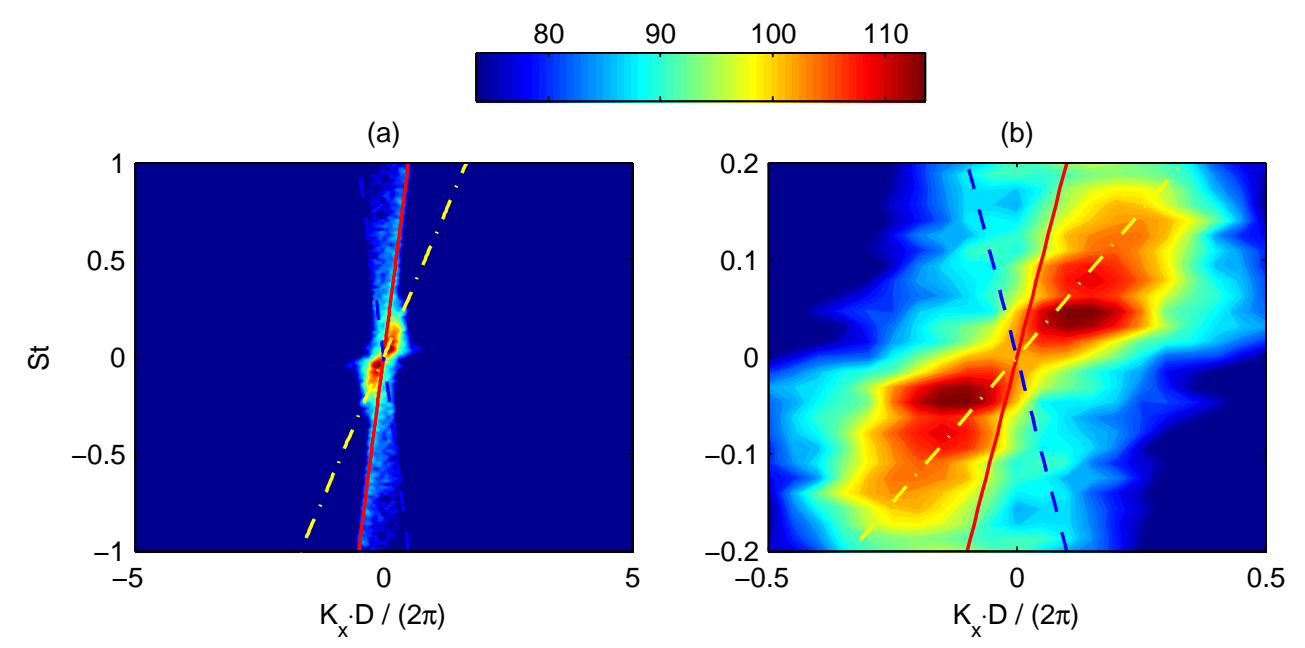

FIGURE 12. The wavenumber spectrum of the near-field pressure along the line $r=3 D, \theta=0$ : (a) full range; (b) zoomed-in central region. The red solid and blue dashed line correspond the convection velocities $U_{c}=c_{0}$ and $U_{c}=-c_{0}$ respectively; the dash-dotted yellow line corresponds to the convection velocity $U_{c}=0.6 U_{j}$.

significantly less than $0.6 U_{j}$ (the convection peaks on the right hand side are below the line of $U_{c}=0.6 U_{j}$ ). It is worth noting that due to the size limitation of the computational domain, the highest spatial resolution we achieved is $k_{x} D /(2 \pi) \approx 0.07$. Therefore at very low frequencies, e.g. $S t<0.03$, the convective peaks cannot be resolved. Also worth noting is that the convection peaks we show in this figure corresponds to an overall convection velocity for the axial range $x=0$ to $14 D$, therefore it is somewhat different from the local convection velocities obtained in figure 11.

\subsubsection{Near-field pressure scattering}

Using the conclusions we made about the near-field pressure in the preceding section, we develop a closed-form scattering model in this section. For a realistic wing-flap system, both the trailing edge and side edges of the wing and flap are present. We again adopt the same simplification used in developing the acoustic Green's function: the wing and flap system are assumed to a semi-infinite flat plate with only a trailing edge. Due to the presence of the flat plate, we switch back to the Cartesian coordinates defined in figure 2 again. When the flat plate is sufficiently far away from the jet axis, for example the perpendicular distance between the plate and the jet axis is greater than $2 D$, little change of the flow occurs due to the presence of the plate and therefore the near-field evanescent waves, originating from hydrodynamic instability waves, can be found to be virtually same as that for an isolated jet (Bychkov \& Faranosov 2014). We can thus use the evanescent wave field for an isolated jet as the incident evanescent field for an installed jet. Assume the near-field pressure fluctuation is dominated by the evanescent wave of the first few modes, i.e. the pressure field can be written as

$$
p^{\prime}(\omega, \boldsymbol{x})=\sum_{m=-N}^{N} \hat{p}(\omega, m) K_{m}(\gamma r) \mathrm{e}^{-\mathrm{i} k_{1} x_{1}} \mathrm{e}^{\mathrm{i} m \theta}
$$

where $\hat{p}(\omega, m)$ denotes the magnitude of the pressure fluctuations of mode $m$ and frequency $\omega, K_{m}$ the $m$-th order modified Bessel function of the second kind and the radius $r$ here is now defined as $\sqrt{x_{2}^{2}+\left(x_{3}+H\right)^{2}}$, where $H$ is the distance between the jet cen- 
tre line to the flat plate. $\gamma$, as defined earlier, denotes the decay rate $\sqrt{k_{1}^{2}-k^{2}}$ where $k_{1}=\omega / U_{c}$. The convection velocity $U_{c}$ (hence $k_{1}$ ) is a function of $\omega$ and $m$. $\theta$ is the azimuthal angle and $N$ is a small integer, for example according to the LES data, we find $N=1$ is sufficient.

However (2.37) is the solution of the reduced wave equation with a static ambient flow. In the presence of an uniform ambient flow of speed $U$ in the $x_{1}$ direction, (2.37) changes to

$$
p^{\prime}(\omega, \boldsymbol{x})=\sum_{m=-N}^{N} \hat{p}(\omega, m) K_{m}\left(\gamma_{c} r\right) \mathrm{e}^{-\mathrm{i} k_{1} x_{1}} \mathrm{e}^{\mathrm{i} m \theta},
$$

where the convective radial decay rate

$$
\gamma_{c}=\frac{\sqrt{\left(k_{1} \beta^{2}+k M\right)^{2}-k^{2}}}{\beta} .
$$

When the flat plate is directly above the jet, the hypothetical incident pressure that would exist on the lower surface of the plate if the plate were absent is

$$
\begin{aligned}
& p_{h}\left(\omega, x_{1}, x_{2}\right)=\sum_{m=-N}^{N} \hat{p}(\omega, m) K_{m}\left(\gamma_{c} \sqrt{x_{2}^{2}+H^{2}}\right) \mathrm{e}^{-\mathrm{i} k_{1} x_{1}} \\
& \left(\sum_{k=0}^{\left[\frac{|m|}{2}\right]} C_{|m|}^{2 k}(-1)^{k} \frac{H^{|m|-2 k} x_{2}^{2 k}}{\sqrt{x_{2}^{2}+H^{2}}|m|}+\mathrm{i} \operatorname{sgn}(m) \sum_{k=0}^{\left[\frac{|m|-1}{2}\right]} C_{|m|}^{2 k+1}(-1)^{k} \frac{H^{|m|-2 k-1} x_{2}^{2 k+1}}{\sqrt{x_{2}^{2}+H^{2}}|m|}\right),
\end{aligned}
$$

where $[x]$ means taking the nearest integer that is not larger than $x$, and $C_{m}^{n}$ is the binomial coefficient, which results from the use of de Moivre's theorem. By making use of Fourier transformation, the hypothetical incident pressure can be expressed as a superposition of a series of plane waves, namely

$$
p_{h}\left(\omega, x_{1}, x_{2}\right)=\sum_{m=-N}^{N} \int_{-\infty}^{\infty} \tilde{p}\left(\omega, k_{2}, m\right) \mathrm{e}^{-\mathrm{i}\left(k_{1} x_{1}+k_{2} x_{2}\right)} \mathrm{d} k_{2},
$$

where

$$
\begin{aligned}
& \tilde{p}\left(\omega, k_{2}, m\right)=\frac{1}{2 \pi} \int_{-\infty}^{\infty} \hat{p}(\omega, m) K_{m}\left(\gamma_{c} \sqrt{x_{2}^{2}+H^{2}}\right) \mathrm{e}^{\mathrm{i} k_{2} x_{2}} \\
& \left(\sum_{k=0}^{\left[\frac{|m|}{2}\right]} C_{|m|}^{2 k}(-1)^{k} \frac{H^{|m|-2 k} x_{2}^{2 k}}{{\sqrt{x_{2}^{2}+H^{2}}}^{|m|}}+\mathrm{i} \operatorname{sgn}(m) \sum_{k=0}^{\left[\frac{|m|-1}{2}\right]} C_{|m|}^{2 k+1}(-1)^{k} \frac{H^{|m|-2 k-1} x_{2}^{2 k+1}}{\sqrt{x_{2}^{2}+H^{2}}|m|}\right) \mathrm{d} x_{2}
\end{aligned}
$$

Note that the real part of (2.39) is an even function of $x_{2}$ while the imaginary part is an odd one. Making use of this property and properties of Fourier Transformation one can find that the $\hat{p}\left(\omega, k_{2}, m\right)$ can be evaluated analytically (Gradshteyn \& Ryzhik 2007):

$$
\begin{aligned}
& \tilde{p}\left(\omega, k_{2}, m\right)=\frac{1}{\sqrt{2 \pi}} \hat{p}(\omega, m) \\
& \left\{\sum_{k=0}^{\left[\frac{|m|}{2}\right]} C_{|m|}^{2 k} H^{-2 k+\frac{1}{2}} \gamma_{c}^{-|m|} \frac{d^{2 k}}{d k_{2}^{2 k}}\left[\left(\gamma_{c}^{2}+k_{2}^{2}\right)^{\frac{1}{2}|m|-\frac{1}{4}} K_{|m|-\frac{1}{2}}\left(H \sqrt{\gamma_{c}^{2}+k_{2}^{2}}\right)\right]-\operatorname{sgn}(m)\right. \\
& \left.\sum_{k=0}^{\left[\frac{|m|-1}{2}\right]} C_{|m|}^{2 k+1} H^{-2 k+\frac{1}{2}} \gamma_{c}^{-|m|} \frac{d^{2 k}}{d k_{2}^{2 k}}\left[k_{2}\left(\gamma_{c}^{2}+k_{2}^{2}\right)^{\frac{1}{2}|m|-\frac{3}{4}} K_{|m|-\frac{3}{2}}\left(H \sqrt{\gamma_{c}^{2}+k_{2}^{2}}\right)\right]\right\}
\end{aligned}
$$


For an incident pressure given by $\tilde{p}\left(\omega, k_{2}, m\right) \mathrm{e}^{-\mathrm{i}\left(k_{1} x_{1}+k_{2} x_{2}\right)}$, the scattered pressure on the lower surface of the flat plate can be found using Schwarzschild technique as (Amiet 1976a; Lyu et al. 2016)

$$
p_{s}=\tilde{p}\left(\omega, k_{2}, m\right) \mathrm{e}^{-\mathrm{i}\left(k_{1} x_{1}+k_{2} x_{2}\right)}\left((1+\mathrm{i}) E_{0}\left(-\mu x_{1}\right)-1\right),
$$

where

$$
\mu=k_{1}+\sqrt{k^{2}-k_{2}^{2} \beta^{2}} / \beta^{2}+k M / \beta^{2}, E_{0}(x)=\int_{0}^{x} \frac{\mathrm{e}^{-\mathrm{i} t}}{\sqrt{2 \pi t}} \mathrm{~d} t .
$$

When the observer is located at $\boldsymbol{x}$, routine application of the theory of Kirchhoff and Curle yields the far-field sound pressure (Amiet 1975; Lyu et al. 2015, 2016)

$$
\begin{aligned}
p_{f}(\omega, \boldsymbol{x})= & (1+\mathrm{i}) \frac{\omega x_{3}}{\pi c_{0} S_{0}^{2}} \mathrm{e}^{\mathrm{i} k\left(M x_{1}-S_{0}\right) / \beta^{2}} \\
& \sum_{m=-N}^{N} \int_{-\infty}^{\infty} \frac{\sin \left[\left(k_{2}-k \frac{x_{2}}{S_{0}}\right) d / 2\right]}{k_{2}-\frac{x_{2}}{S_{0}}} \frac{1}{\mu_{A}} \Gamma\left(c, \mu, \mu_{A}\right) \tilde{p}\left(\omega, k_{2}, m\right) \mathrm{d} k_{2}
\end{aligned}
$$

where $c$ and $d$ are the chord and span of the finite plate respectively. Note when calculating (2.43) we assumed that the flat-plate is semi-infinite. But when applying the theory of Kirchhoff and Curle to obtain (2.45) we used a finite plate of chord $c$ and span $d$. The same approach was used by Amiet $(1976 b)$ and was found to be a good approximation provided the frequency is not too low (Roger \& Moreau 2005). The properly bounded function $\Gamma$ in $(2.45)$ is defined as

$$
\Gamma\left(x, \mu, \mu_{A}\right)=\mathrm{e}^{\mathrm{i} \mu_{A} x} E_{0}(\mu x)-\sqrt{\frac{\mu}{\mu-\mu_{A}}} E_{0}\left[\left(\mu-\mu_{A}\right) x\right]+\frac{1}{1+\mathrm{i}}\left(1-\mathrm{e}^{\mathrm{i} \mu_{A} x}\right),
$$

and

$$
\begin{aligned}
S_{0} & =\sqrt{x_{1}^{2}+\beta^{2}\left(x_{2}^{2}+x_{3}^{2}\right)}, \\
\mu_{A} & =k_{1}+\frac{k}{\beta^{2}}\left(M-\frac{x_{1}}{S_{0}}\right) .
\end{aligned}
$$

We note that normally aircraft wings have a large span-to-chord ratio, and $d$ can be quite large compared to the sound wavelength at the frequency of peak noise enhancement in the low frequency regime, therefore we use

$$
\lim _{d \rightarrow \infty} \frac{\sin \left[\left(k_{2}-k \frac{x_{2}}{S_{0}}\right) d / 2\right]}{\pi\left(k_{2}-k \frac{x_{2}}{S_{0}}\right)}=\delta\left(k_{2}-k \frac{x_{2}}{S_{0}}\right)
$$

to simplify $(2.45)$ to

$$
p_{f}(\omega, \boldsymbol{x})=(1+\mathrm{i}) \frac{\omega x_{3}}{c_{0} S_{0}^{2}} \sum_{m=-N}^{N} \frac{1}{\mu_{A}} \Gamma\left(c,\left.\mu\right|_{k_{2}=k \frac{x_{2}}{S_{0}}}, \mu_{A}\right) \tilde{p}\left(\omega, k \frac{x_{2}}{S_{0}}, m\right) .
$$

The far-field sound power density spectrum can thus be obtained from $\Phi_{N}(\omega, \boldsymbol{x})=$ $\lim _{T \rightarrow \infty}(\pi / T) \overline{p_{f}(\omega, x), p_{f}^{*}(\omega, x)}$, i.e.:

$$
\begin{aligned}
& \Phi_{N}(\omega, \boldsymbol{x})=\left.\left.2\left[\frac{\omega x_{3}}{c_{0} S_{0}^{2}}\right]^{2} \sum_{m=-N}^{N} \sum_{m^{\prime}=-N}^{N} \frac{\Gamma\left(c,\left.\mu\right|_{k_{2}=k \frac{x_{2}}{S_{0}}}, \mu_{A}\right)}{\mu_{A}}\right|_{m} \frac{\Gamma^{*}\left(c,\left.\mu\right|_{k_{2}=k \frac{x_{2}}{S_{0}}}, \mu_{A}\right)}{\mu_{A}^{*}}\right|_{m^{\prime}} \\
& \lim _{T \rightarrow \infty} \frac{\pi}{T} \overline{\tilde{p}\left(\omega, k \frac{x_{2}}{S_{0}}, m\right) \tilde{p}^{*}\left(\omega, k \frac{x_{2}}{S_{0}}, m^{\prime}\right)}
\end{aligned}
$$

It should be noted that $(2.50)$ is due to the contribution of the scattered pressure only. 
In order to take the incident wave contribution into consideration, the term 1 in the last bracket on the right hand side of (2.46) defining the function $\Gamma\left(x, \mu, \mu_{A}\right)$ should be omitted (Amiet 1978).

Substituting (2.42) into the above equation, one finds the statistical term in (2.50) can be evaluated to be

$$
\begin{aligned}
& \lim _{T \rightarrow \infty} \frac{\pi}{T} \overline{\tilde{p}\left(\omega, k \frac{x_{2}}{S_{0}}, m\right) \tilde{p}^{*}\left(\omega, k \frac{x_{2}}{S_{0}}, m^{\prime}\right)}=\frac{1}{2 \pi} \Pi(\omega, m) \delta_{m m^{\prime}} \\
& \times\left\{\sum_{k=0}^{\left[\frac{|m|}{2}\right]} C_{|m|}^{2 k} H^{-2 k+\frac{1}{2}} \gamma_{c}^{-|m|} \frac{d^{2 k}}{d k_{2}^{2 k}}\left[\left(\gamma_{c}^{2}+k_{2}^{2}\right)^{\frac{1}{2}|m|-\frac{1}{4}} K_{|m|-\frac{1}{2}}\left(H \sqrt{\gamma_{c}^{2}+k_{2}^{2}}\right)\right]-\operatorname{sgn}(m) \times\right. \\
& \left.\sum_{k=0}^{\left[\frac{|m|-1}{2}\right]} C_{|m|}^{2 k+1} H^{-2 k+\frac{1}{2}} \gamma_{c}^{-|m|} \frac{d^{2 k}}{d k_{2}^{2 k}}\left[k_{2}\left(\gamma_{c}^{2}+k_{2}^{2}\right)^{\frac{1}{2}|m|-\frac{3}{4}} K_{|m|-\frac{3}{2}}\left(H \sqrt{\gamma_{c}^{2}+k_{2}^{2}}\right)\right]\right\}_{k_{2}=\frac{k x_{2}}{S 0}}^{2}
\end{aligned}
$$

where $\Pi(\omega, m)$ denotes the power spectrum of $m$-th order near-field evanescent waves. The spectrum could be obtained from simple models validated by experiments or LES simulations. Substituting (2.51) into (2.50) yields

$$
\begin{aligned}
& \Phi_{N}(\omega, \boldsymbol{x})=\frac{1}{\pi}\left[\frac{\omega x_{3}}{c_{0} S_{0}^{2}}\right]^{2} \sum_{m=-N}^{N}\left|\frac{\Gamma\left(c,\left.\mu\right|_{k_{2}=k \frac{x_{2}}{S_{0}}, \mu_{A}}\right)}{\mu_{A}}\right|^{2} \Pi(\omega, m) \\
& \times\left\{\sum_{k=0}^{\left[\frac{|m|}{2}\right]} C_{|m|}^{2 k} H^{-2 k+\frac{1}{2}} \gamma_{c}^{-|m|} \frac{d^{2 k}}{d k_{2}^{2 k}}\left[\left(\gamma_{c}^{2}+k_{2}^{2}\right)^{\frac{1}{2}|m|-\frac{1}{4}} K_{|m|-\frac{1}{2}}\left(H \sqrt{\gamma_{c}^{2}+k_{2}^{2}}\right)\right]-\operatorname{sgn}(m) \sum_{k=0}^{\left[\frac{|m|-1}{2}\right]}\right. \\
& \left.C_{|m|}^{2 k+1} H^{-2 k+\frac{1}{2}} \gamma_{c}^{-|m|} \frac{d^{2 k}}{d k_{2}^{2 k}}\left[k_{2}\left(\gamma_{c}^{2}+k_{2}^{2}\right)^{\frac{1}{2}|m|-\frac{3}{4}} K_{|m|-\frac{3}{2}}\left(H \sqrt{\gamma_{c}^{2}+k_{2}^{2}}\right)\right]\right\}_{k_{2}=\frac{k x_{2}}{S 0}}^{2} \cdot(2.52)
\end{aligned}
$$

Equation (2.52) is the generic form of near-field scattering model. However, further simplifications can be made in practical cases. First if we assume that the fluctuation is symmetric with respect to $m$, i.e. $\Pi(\omega, m)=\Pi(\omega,-m),(2.52)$ can be further simplified to

$$
\begin{aligned}
& \Phi_{N}(\omega, \boldsymbol{x})=\frac{1}{\pi}\left[\frac{\omega x_{3}}{c_{0} S_{0}^{2}}\right]^{2} \sum_{m=0}^{N}\left|\frac{\Gamma\left(c,\left.\mu\right|_{k_{2}=k \frac{x_{2}}{S_{0}}}, \mu_{A}\right)}{\mu_{A}}\right|^{2} \Pi_{s}(\omega, m) \\
& \times\left\{\left(\sum_{k=0}^{\left[\frac{|m|}{2}\right]} C_{|m|}^{2 k} H^{-2 k+\frac{1}{2}} \gamma_{c}^{-|m|} \frac{d^{2 k}}{d k_{2}^{2 k}}\left[\left(\gamma_{c}^{2}+k_{2}^{2}\right)^{\frac{1}{2}|m|-\frac{1}{4}} K_{|m|-\frac{1}{2}}\left(H \sqrt{\gamma_{c}^{2}+k_{2}^{2}}\right)\right]\right)^{2}+\left(\sum_{k=0}^{\left[\frac{|m|-1}{2}\right]}\right.\right. \\
& \left.\left.C_{|m|}^{2 k+1} H^{-2 k+\frac{1}{2}} \gamma_{c}^{-|m|} \frac{d^{2 k}}{d k_{2}^{2 k}}\left[k_{2}\left(\gamma_{c}^{2}+k_{2}^{2}\right)^{\frac{1}{2}|m|-\frac{3}{4}} K_{|m|-\frac{3}{2}}\left(H \sqrt{\gamma_{c}^{2}+k_{2}^{2}}\right)\right]\right)^{2}\right\}_{k_{2}=\frac{k x_{2}}{S 0}} \cdot(2.53)
\end{aligned}
$$

where $\Pi_{s}(\omega, m)$ is $m$-th single-sided modal power spectral density, i.e. $\Pi_{s}(\omega, m)=$ $\Pi(\omega, m)+\Pi(\omega,-m)$ for $m \neq 0$. If we assume that only the 0 and $1(-1)$ modes are significant, we can show that the far-field sound spectral density in the mid-span plane 
$\left(x_{2}=0\right)$ is

$$
\begin{aligned}
\Phi_{N}(\omega, \boldsymbol{x}) \approx & {\left[\frac{\omega x_{3}}{c_{0} S_{0}^{2}}\right]^{2}\left\{\left|\frac{\Gamma\left(c, \mu, \mu_{A}\right)}{\mu_{A}}\right|^{2} \Pi_{s}(\omega, 0) \frac{\mathrm{e}^{-2 H \gamma_{c}}}{2 \gamma_{c}^{2}}\right\}_{k_{2}=0, m=0} } \\
& +\left\{\left|\frac{\Gamma\left(c, \mu, \mu_{A}\right)}{\mu_{A}}\right|^{2} \Pi_{s}(\omega, 1) \frac{\mathrm{e}^{-2 H \gamma_{c}}}{2 \gamma_{c}^{2}}\right\}_{k_{2}=0, m=1} .
\end{aligned}
$$

Let $\Pi_{0}(\omega, 0)$ and $\Pi_{0}(\omega, 1)$ denote the 0 -th and 1-st single-sided modal power spectral densities measured at the location of $r=r_{0}$, then it follows that $\Pi_{s}(\omega, 0) K_{0}^{2}\left(\gamma_{c} r_{0}\right)=$ $\Pi_{0}(\omega, 0)$ and $\Pi_{s}(\omega, 1) K_{1}^{2}\left(\gamma_{c} r_{0}\right)=\Pi_{0}(\omega, 1)$. Note in $(2.54), \mu, \mu_{A}$ and $\gamma_{c}$ depend on the mode number $m$ as the convection velocity (hence $k_{1}$ ) can vary with $m$. However in section 2.2.1 we show that the convection velocity for mode 0 and $( \pm) 1$ do not differ significantly from each other, therefore (2.54) can be further simplified by assuming an averaged convection velocity profile $\bar{U}_{c}(\omega)$ over the two modes, such that

$\Phi_{N}(\omega, \boldsymbol{x}) \approx\left[\frac{\omega x_{3}}{c_{0} S_{0}^{2}}\right]^{2}\left\{\left|\frac{\Gamma\left(c, \mu, \mu_{A}\right)}{\mu_{A}}\right|^{2} \frac{\mathrm{e}^{-2 H \gamma_{c}}}{2 \gamma_{c}^{2}}\left(\frac{\Pi_{0}(\omega, 0)}{K_{0}^{2}\left(\gamma_{c} r_{0}\right)}+\frac{\Pi_{0}(\omega, 1)}{K_{1}^{2}\left(\gamma_{c} r_{0}\right)}\right)\right\}_{k_{2}=0, U_{c}=\bar{U}_{c}(\omega)}$,

When in the frequency of interests where $K_{0}\left(\gamma_{c} r_{0}\right)$ and $K_{1}\left(\gamma_{c} r_{0}\right)$ do not differ from each other significantly (such as the case to be presented in the section 3), (2.55) can be estimated by

$$
\Phi_{N}(\omega, \boldsymbol{x}) \approx\left[\frac{\omega x_{3}}{c_{0} S_{0}^{2}}\right]^{2}\left\{\left|\frac{\Gamma\left(c, \mu, \mu_{A}\right)}{\mu_{A}}\right|^{2} \frac{\mathrm{e}^{-2 H \gamma_{c}}}{2 \gamma_{c}^{2}} \frac{\Pi_{0}(\omega)}{K_{0}^{2}\left(\gamma_{c} r_{0}\right)}\right\}_{k_{2}=0, U_{c}=\bar{U}_{c}(\omega)},
$$

where $\Pi_{0}(\omega)$ is the single-sided spectrum of the incident near-field evanescent waves at $r=r_{0}$, which can be easily measured using one microphone in an isolated-jet experiment. To ensure acoustic fluctuations to be negligible, one can choose $r_{0}$ to be small such that the microphone is sufficiently close to the jet. Since this spectrum varies with axial position, it makes more sense to put the microphone at the position where the trailing edge of the flat plate would be if a flat plate were to be present, i.e. the place where the incident wave is to be scattered.

We have now obtained the far-field sound due to the quadrupole sources, i.e. $\Phi_{Q}(\boldsymbol{x}, \omega)$ as shown in (2.18), and the sound due to the interaction between the near-field evanescent wave and the trailing edge of the flat plate, i.e. $\Phi_{N}(\boldsymbol{x}, \omega)$ as shown from (2.52) to (2.56), the total sound power spectra is therefore

$$
\Phi(\boldsymbol{x}, \omega)=\Phi_{Q}(\boldsymbol{x}, \omega)+\Phi_{N}(\boldsymbol{x}, \omega) .
$$

It is useful to review the inputs of this model. First, $\Phi_{Q}(\boldsymbol{x}, \omega)$ is the sound predicted using Lighthill's acoustic analogy. The Green's function is obtained analytically and therefore the input of this part is the time-averaged flow statistics obtained from RANS. Secondly, the near-field scattering part, e.g. $\Phi_{N}(\boldsymbol{x}, \omega)$ shown in $(2.56)$, requires the onepoint spectrum and the local convection velocity (varying with frequency as well) of the near-field pressure fluctuation of an isolated jet at the location where the trailing edge of the flat plate would be. It is worth mentioning that though we perform an LES study in this paper, it is mostly for validation purposes, and the hybrid model does not directly require the input of the LES (at least for the cases the where convection velocity curve still applies or is known from experiments or models of instability waves).

It is worth noting that though the new model requires the near-field pressure spectrum as an input, this requirement could be removed if the strength of the evanescent instability 


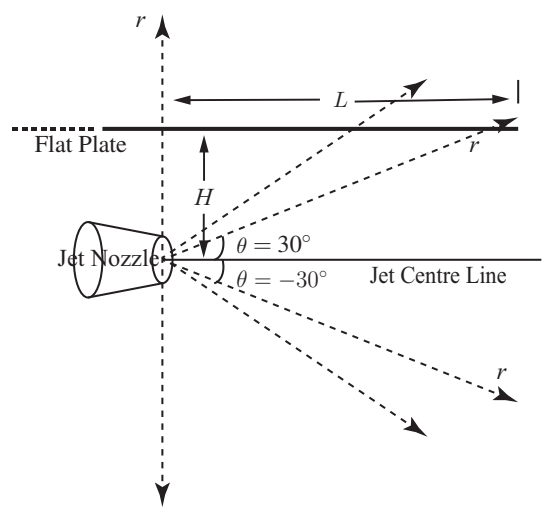

Figure 13. The schematic of the experiment of Head \& Fisher (1976). The separation distance between the flat plate and the jet centreline $H=3 D$, the distance between the trailing edge of the flat plate and the jet nozzle $L=6 D$.

waves were predicted by modelling the growth and decay of the jet instability waves, as is common in instability theory. However to apply that to a particular experiment requires data on the perturbations at the nozzle exit. That information was not available in the experiment of Head \& Fisher (1976), but the near-field pressure was and we have used that for validation. Therefore, in general, a prediction model independent of experimental measurement is possible for specified exit boundary conditions. Though we do not have that information about boundary conditions to follow this approach, the semi-analytical model developed in the paper is also very useful in both understanding the underlying physics and developing ideas to control it. For example the near-field scattering model proposed in this paper is nothing more than a transfer function, which maps the input (near-field pressure) spectrum to the output (far-field sound) spectrum. The fact it works well (as shown in the next section) confirms that the scattering mechanism of the installed jet noise, and therefore provides evidence of this new sound source as opposed to isolated jets. By studying this transfer function, we can also understand the transfer efficiency, i.e. at which frequency the scattering is most efficient. The identification of the sound source and the understanding of the scattering mechanism is essential for noise reduction methods.

It should also be noted that while the ambient flow is taken into consideration and the effect of source motion is accounted for by the model of the fourth-order space-time correlation function, the model for installed jet noise developed in this section does not account for the mean jet flow refraction effect. Though this means that the model cannot correctly predict the far-field sound at high frequencies, it should suffice for the sound at $90^{\circ}$ to the jet and for low frequencies. In the next section, we will use the model developed in this section to predict the installed jet noise spectrum and the results will be compared against experimental measurements.

\section{Results}

In 1976, in order to study the acoustic characteristics of low frequency enhancement of the installed jet noise and to identify the corresponding noise sources, Head \& Fisher (1976) presented a series of experimental results of the low frequency augmentation of the jet noise with the close presence of a solid shield (flat plate), as shown in figure 13. There was no ambient flow so $M=0$. The experiment was carried out with a cold, subsonic, round jet ( $D=1$ inch) at $M_{0}=0.5$ and the separation distance between the 
plate and the jet centreline $H=3 D$ and the distance between the trailing edge of the flat plate and the jet nozzle $L=6 D$. The far-field spectra for both isolated and installed jet noise were measured and the microphone was located in the plane that is perpendicular to the rigid shield but at $90^{\circ}, 45^{\circ}$ and $30^{\circ}$ to the jet centreline respectively, on both the shielded and reflected sides. Since the rigid shield used in the experiment was placed sufficiently away from the jet, it can be expected to have little effect on the jet flow. Thus, in this section we use the two-part model, together with the inputs from axisymmetric jet flow field obtained in the preceding section, to calculate both the isolated and installed jet noise spectra. In the Lighthill acoustic analogy part, $\Phi_{Q}(\boldsymbol{x}, \omega)$, the turbulent statistics is obtained from the RANS calculation. In the near-field scattering part, we use the approximated (2.56) to calculate $\Phi_{N}(\boldsymbol{x}, \omega)$, and the spectrum of the near-field evanescent waves at the trailing-edge position was experimentally measured. But the local convection velocity at this point makes use of the averaged frequencydependent velocity obtained from the aforementioned LES study. The results based on the new model are then compared with the experimental results.

\subsection{Installed jet with acoustic scattering of Lighthill's quadrupole sources}

To demonstrate the existence of the additional near-field scattering mechanism for installed jet noise, the prediction of the far-field sound power spectrum with only the contribution of Lighthill's quadrupole sources, i.e. $\Phi_{Q}(\boldsymbol{x}, \omega)$, is presented first. The predictions are compared with the experimental results.

Figure 14(a) shows the sound power spectra at $90^{\circ}$ to the jet on the shielded side. The isolated sound spectra are also presented. As can be seen, the predicted spectrum for an isolated jet has an excellent agreement with experimental results. This shows that the RANS calculation and the fourth-order correlation model indeed work well. However the predicted spectrum for an installed jet does not agree well with the experimental result. In particular, the predicted spectrum fails to capture the low frequency amplification. The reason is because we have not yet incorporated the near-field scattering mechanism, which will be shown to account for such a noise intensification. The discrepancy at high frequencies, however, is in fact expected, since the jet refraction effect must be accounted for in order to correctly predict the shielding effect of the flat plate.

The noise spectra at $45^{\circ}$ and $30^{\circ}$ to the jet are shown in figures $14(\mathrm{~b})$ and $14(\mathrm{c})$, respectively. The predicted spectra for isolated jet noise continues to agree well with experiment at low frequencies. But discrepancies start to appear at high frequencies, because the jet mean-flow refraction effect cannot be ignored at these angles. For the installed jet noise, the low frequency enhancement at low frequencies at $45^{\circ}$ still fails to be captured and the agreement at high frequencies is also affected by the refraction effect of the jet mean flow. But the prediction does indeed give some of the correct qualitative behaviour.

Figure 14(d) shows the noise power spectra predicted at $90^{\circ}$ on the reflected side. For installed jet noise spectra, despite the low frequency discrepancies, the agreement at high frequencies is in fact very good. This indicates that the high frequency reflection effect can be correctly captured at $90^{\circ}$ to the jet. Figures $14(\mathrm{e})$ and $14(\mathrm{f})$ present the noise spectra at $45^{\circ}$ and $30^{\circ}$ respectively. For installed jet noise, despite the large discrepancies caused by the jet refraction effect, it is found that the qualitative behaviour of the noise increase due to the flat plate observed at high frequencies is correctly predicted. This suggests that if the refraction effect were to be included, the model would be able to give much better agreement with the experiment at high frequencies. It is worth noting that, at all the different observer angles, the low frequency amplification cannot be captured, and 


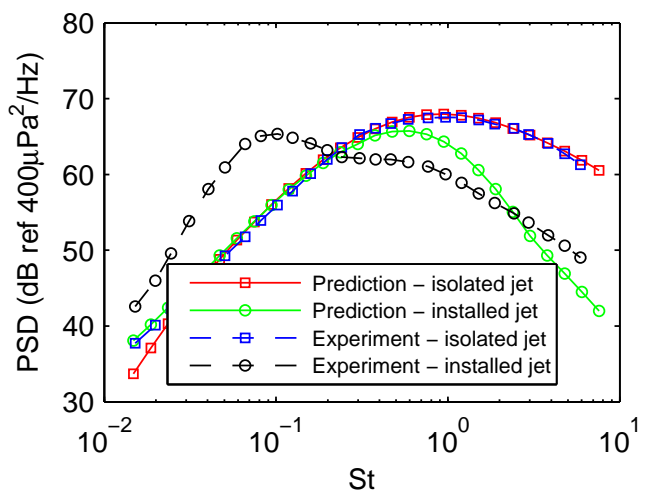

(a) $90^{\circ}$ to the jet, shielded side

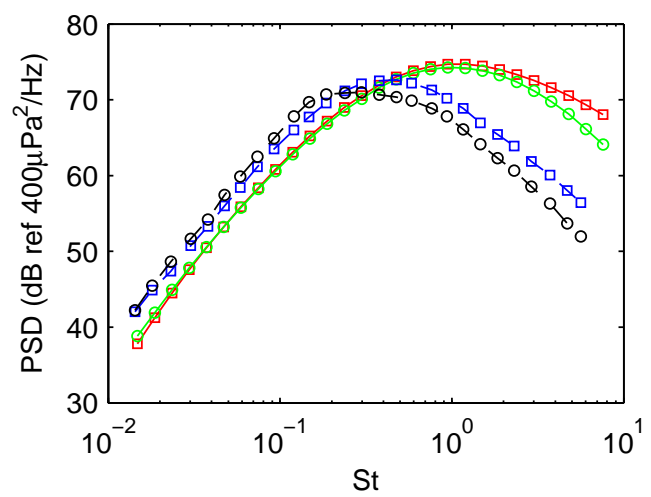

(c) $30^{\circ}$ to the jet, shielded side

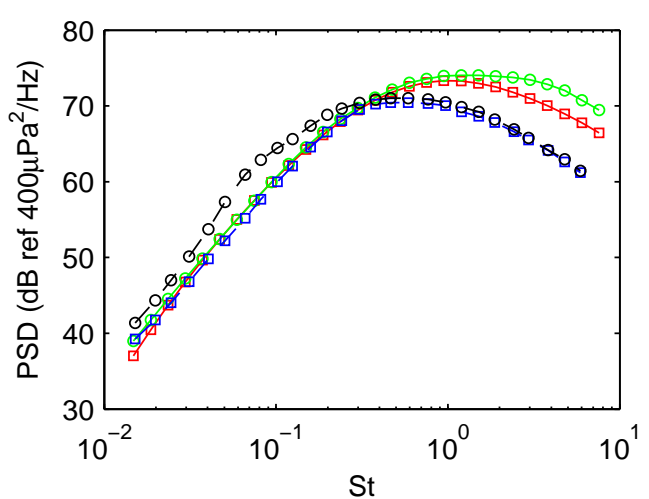

(e) $45^{\circ}$ to the jet, reflected side

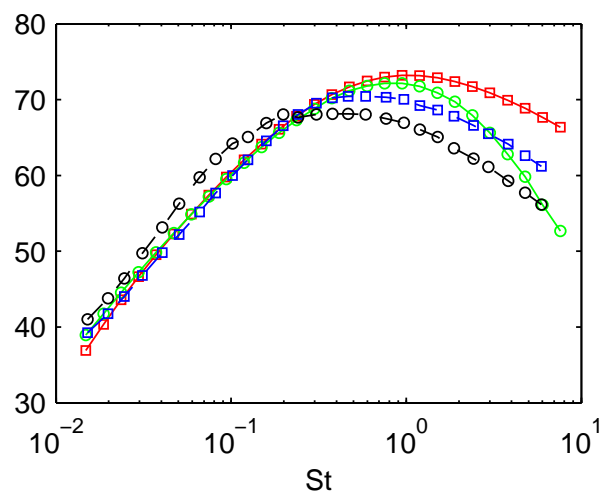

(b) $45^{\circ}$ to the jet, shielded side

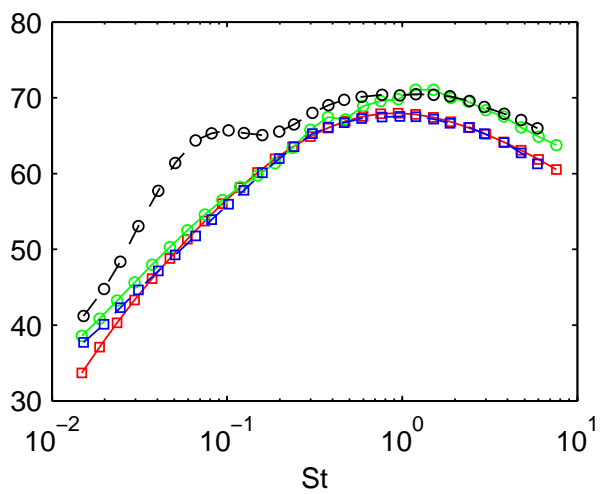

(d) $90^{\circ}$ to the jet, reflected side

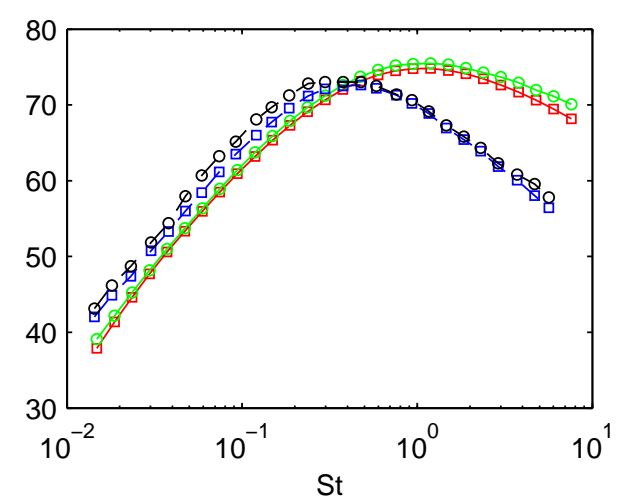

(f) $30^{\circ}$ to the jet, reflected side

Figure 14. Comparison between experimental measurements with model predictions for the far-field sound power spectrum in $1 / 3$ octaves for the installed jet with only the contribution of Lighthill's quadrupole sources.

this suggests that the near-field scattering mechanism is responsible for the low frequency noise alteration. 


\subsection{Installed noise with two source mechanisms}

From figures 14(a) to 14(f), it has been found that the noise spectrum predicted by incorporating only the contribution of the scattering of the Lighthill's quadrupole sources cannot correctly model installed jet noise. In this part of this section, the contribution of the near-field scattering, i.e. $\Phi_{N}(\boldsymbol{x}, \omega)$ described in Section 2.2, is added. As mentioned above, the convection velocity of the near-field evanescent wave obtained by examining the radial decay rate using the LES data is used. We use the averaged convection velocity profile $\bar{U}_{c}(\omega)$ obtained by averaging over modes 0 and $( \pm) 1$. By combining the frequency-dependent convection velocity $\bar{U}_{c}(\omega)$ and the experimentally measured nearfield spectrum, the predicted far-field sound spectra at different angles are compared with experiments.

The sound spectra for both isolated and installed jets on the shielded side are shown in figures $15(\mathrm{a})$ to $15(\mathrm{c})$. As the spectra for isolated jet is identical to those shown in figure 14, we will only focus on the installed spectra hereafter. The predicted noise spectra at $90^{\circ}$ to the jet on the shielded side is presented in figure $15(\mathrm{a})$. It can be seen that very good agreement at low frequencies was achieved between the predicted and experimental results. A few reasons can be guessed to account for the slight under-prediction near the peak frequencies. First it can be due to the slight error of estimated convection velocity since the scattering is highly sensitive to this quantity. An experimental error is also possible: since the near-field pressure decays exponentially in the radial direction, a small misalignment of $2 \mathrm{~mm}$ for example, can cause a PSD change of up to $2 \mathrm{~dB}$. Given the solid shield used in the experiment can easily have a thickness of $2 \mathrm{~mm}$, the error could have been easily introduced. The sound spectra predicted at $45^{\circ}$ to the jet are shown in figure 15(b). Comparing with figure 14(b), where not all the low frequency enhancement is captured, figure 15(b) does indeed better agree with the experimental results at low frequencies. Similarly, the sound spectra at $30^{\circ}$ is shown in figure $15(\mathrm{c})$, and the agreement with experimental results is good. As mentioned in the preceding subsection, the high frequency sound is not predicted quantitatively due to the mean flow refraction effect, but the qualitative behaviours are successfully captured.

Figures $15(\mathrm{~d})$ to $15(\mathrm{f})$ show the comparisons on the reflected side. As can be seen from figure $15(\mathrm{~d})$, the agreement is similar to that at $90^{\circ}$ on the shielded side. The low frequencies enhancement is dominated by the near-field scattering while the high frequency amplification is due to the pure reflection effect which is correctly captured using Lighthill's quadrupole sources. The agreement at $45^{\circ}$ and $30^{\circ}$ to the jet is very similar to that on the shielded side. In particular, at $45^{\circ}$ the model prediction agrees better with experimental results. The high frequency deviation, as already mentioned for the isolated jet, is caused by the refraction effect of the jet mean flow.

Even though overall good agreement between the model predictions and the experiment results at different observer angles is achieved at low frequencies, it should be noted that the high frequency sound at low observer angles cannot be predicted correctly due to the jet refraction effect. A more accurate model aiming to include this effect is desired, and will form part of our future work.

\section{Conclusion}

This paper develops a low-order model to predict installed jet noise. The model starts with the development of a half-plane scattering Green's function. The Green's function is then used to solve the Lighthill's acoustic analogy equation together with a model for the Lighthill's quadrupole sources and the mean flow data obtained by performing RANS 


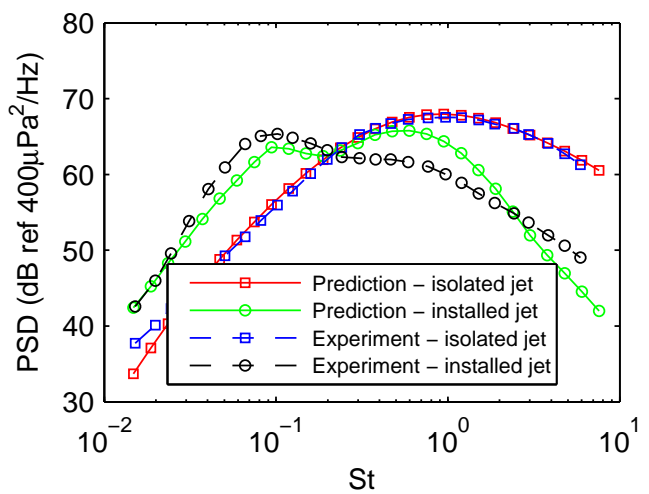

(a) $90^{\circ}$ to the jet, shielded side

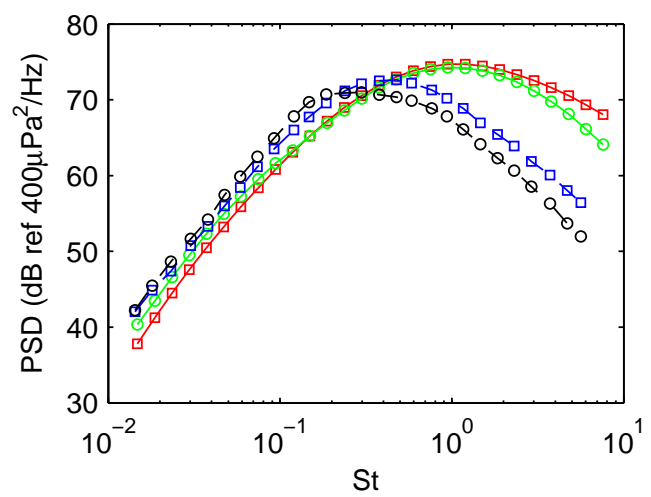

(c) $30^{\circ}$ to the jet, shielded side

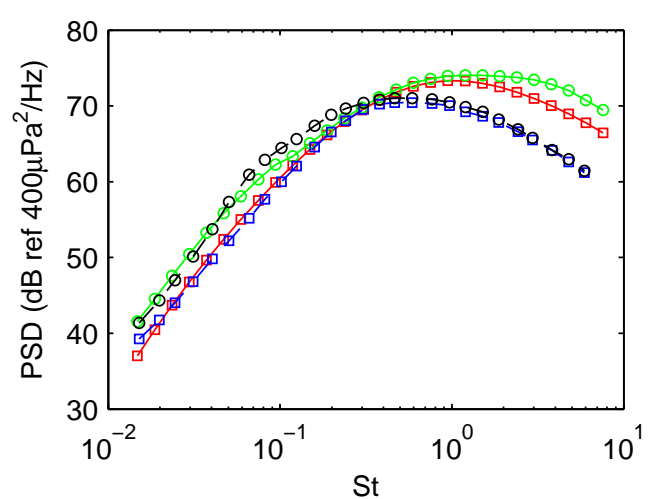

(e) $45^{\circ}$ to the jet, reflected side

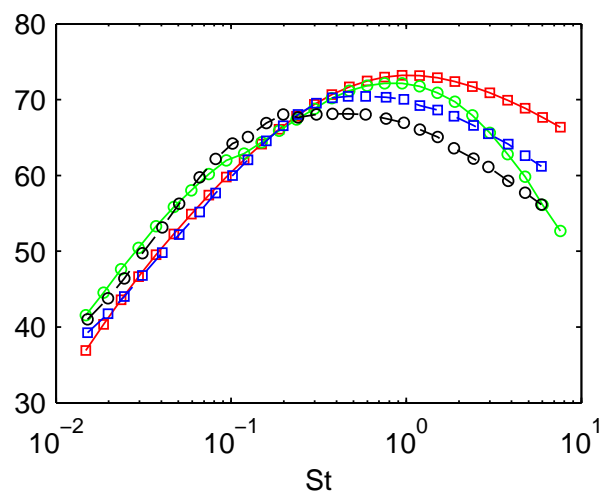

(b) $45^{\circ}$ to the jet, shielded side

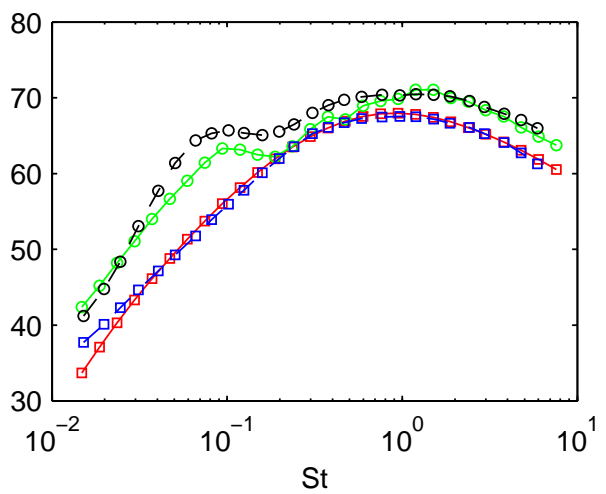

(d) $90^{\circ}$ to the jet, reflected side

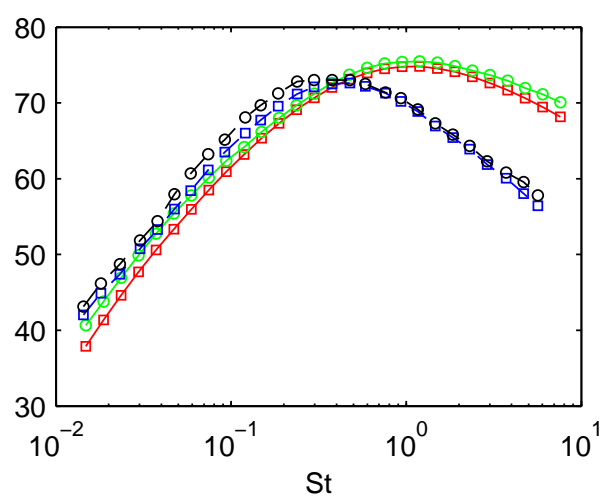

(f) $30^{\circ}$ to the jet, reflected side

Figure 15. Comparison between experimental measurements with model predictions for the far-field sound power spectrum in $1 / 3$ octaves for the installed jet with two source mechanisms

calculations. The additional sound source due to near-field evanescent wave scattering is accounted for by developing a trailing-edge scattering model using Amiet's approach. Therefore, the resulting far-field sound consists of the sum of contributions from two 
source mechanisms: Lighthill's quadrupole sources and the near-field evanescent wave scattering. Finally, the proposed model is validated against experimental measurements.

It is found that when a solid shield (either a wing or a flat plate) is sufficiently far away from the jet, e.g. when the perpendicular distance between the shield and jet axis $H$ is greater than $2 D$, the noise at low frequencies $(S t<0.2)$ due to Lighthill's quadrupole sources is hardly affected by the presence of the solid shield. For high frequencies, however, the far-field sound due to quadrupole sources is either efficiently shielded at $90^{\circ}$ to the jet on the shielded side or enhanced by around $3 \mathrm{~dB}$ at $90^{\circ}$ to the jet on the reflected side. The sound from the quadrupole sources is also significantly refracted by the jet mean flow for the $M_{0}=0.5$ jet considered. Thus at low observer angles where the refraction effect is significant, only qualitatively agreement is achieved.

It is shown that the significant low frequency noise enhancement observed in installed jet experiments is due to the interaction between the near-field evanescent wave and the trailing edge of the solid shield near the jet. The evanescent wave is believed to originate from the Kelvin-Helmholtz instability mechanism. Using the averaged $\bar{U}_{c}(\omega)$ obtained from LES and the measured near-field evanescent pressure spectrum at the position where the trailing edge of the flat plate would be, the near-field scattering model can successfully predict the noise spectra at various observer angles. The sound due to near-field scattering is however only significant at low frequencies and negligible at high frequencies.

As already mentioned, one limitation of the two-part model developed in this paper is that it does not account for the jet refraction effect which is significant at low observer angels to the jet for high frequency sound and non-negligible jet Mach numbers. Thus even though the qualitative behaviour of the far-field sound at high frequencies due to the presence of the flat shield is successfully predicted, quantitative agreement is not achieved except for the spectrum at $90^{\circ}$ to the jet on the reflected side. Another limitation is that the solid shield is assumed to be semi-infinite in the proposed model, and thus the effect of its side edges is ignored.

\section{Acknowledgement}

The first author (BL) wishes to gratefully acknowledge the financial support co-funded by the Cambridge Commonwealth European and International Trust and the China Scholarship Council. The third author (IN) wishes to acknowledge the UK Turbulence Consortium (UKTC) for the high performance computing time to carry out the LES simulation on ARCHER under EPSRC grant number EP/L000261/1 and under PRACE award on HERMIT.

\section{Appendix}

Detailed expression for the directivity terms $D_{i j}^{k}$ are shown as follows,

$$
\begin{aligned}
& D_{12}^{0}\left(y_{1}, y_{3}\right)=-\frac{\cos \bar{\theta}(-M+\cos \bar{\alpha})}{\beta^{3}}\left(E\left(u_{R}\right) \mathrm{e}^{\mathrm{i} \frac{k}{\beta} \cos \bar{\gamma} y_{3}}+E\left(u_{R^{\prime}}\right) \mathrm{e}^{-\mathrm{i} \frac{k}{\beta} \cos \bar{\gamma} y_{3}}\right), \\
& D_{12}^{1}\left(y_{1}, y_{3}\right)=-\frac{\mathrm{i} \cos \bar{\theta} \mathrm{i}^{\mathrm{i} \frac{\pi}{4}} \sqrt{\sin \bar{\theta}}}{\beta^{2} \sqrt{2 \pi}}
\end{aligned}
$$

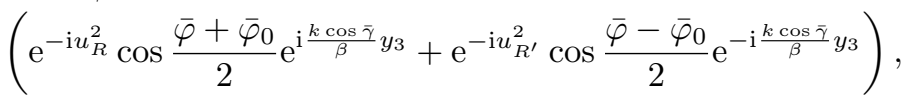




$$
\begin{aligned}
D_{23}^{0}\left(y_{1}, y_{3}\right)= & \frac{-\cos \bar{\theta} \cos \bar{\gamma}}{\beta^{2}}\left(E\left(u_{R}\right) \mathrm{e}^{\mathrm{i} \frac{k}{\beta} \cos \bar{\gamma} y_{3}}-E\left(u_{R^{\prime}}\right) \mathrm{e}^{-\mathrm{i} \frac{k}{\beta} \cos \bar{\gamma} y_{3}}\right), \\
D_{23}^{1}\left(y_{1}, y_{3}\right)= & \frac{\mathrm{i} \cos \bar{\theta} \mathrm{e}^{\mathrm{i} \frac{\pi}{4}} \sqrt{\sin \bar{\theta}}}{\beta \sqrt{2 \pi}} \\
& \left(\mathrm{e}^{-\mathrm{i} u_{R}^{2}} \sin \frac{\bar{\varphi}+\bar{\varphi}_{0}}{2} \mathrm{e}^{\mathrm{i} \frac{k \cos \bar{\gamma}}{\beta}} y_{3}-\mathrm{e}^{-\mathrm{i} u_{R^{\prime}}^{2}} \sin \frac{\bar{\varphi}-\bar{\varphi}_{0}}{2} \mathrm{e}^{-\mathrm{i} \frac{k \cos \bar{\gamma}}{\beta} y_{3}}\right),
\end{aligned}
$$

$$
\begin{aligned}
& D_{11}^{0}\left(y_{1}, y_{3}\right)=-\left(\frac{-M+\cos \bar{\alpha}}{\beta^{2}}\right)^{2}\left(E\left(u_{R}\right) \mathrm{e}^{\mathrm{i} \frac{k}{\beta} \cos \bar{\gamma} y_{3}}+E\left(u_{R^{\prime}}\right) \mathrm{e}^{-\mathrm{i} \frac{k}{\beta} \cos \bar{\gamma} y_{3}}\right), \\
& D_{11}^{1}\left(y_{1}, y_{3}\right)=-\frac{2 \mathrm{i}(-M+\cos \alpha) \mathrm{e}^{\mathrm{i} \frac{\pi}{4}} \sqrt{\sin \bar{\theta}}}{\beta^{3} \sqrt{2 \pi}}\left(\mathrm{e}^{-\mathrm{i} u_{R}^{2}} \cos \frac{\bar{\varphi}+\bar{\varphi}_{0}}{2} \mathrm{e}^{\mathrm{i} \frac{k \cos \bar{\gamma}}{\beta} y_{3}}\right. \\
& \left(1+\frac{\sin \bar{\theta}}{(-M+\cos \bar{\alpha})} \cos \left(\frac{\bar{\varphi}+\bar{\varphi}_{0}}{2}\right) \cos \left(\frac{\bar{\varphi}-\bar{\varphi}_{0}}{2}\right)\right) \\
& +\mathrm{e}^{-\mathrm{i} u_{R^{\prime}}^{2}} \cos \frac{\bar{\varphi}-\bar{\varphi}_{0}}{2} \mathrm{e}^{-\mathrm{i} \frac{k \cos \bar{\gamma}}{\beta} y_{3}} \\
& \left.\left(1+\frac{\sin \bar{\theta}}{(-M+\cos \bar{\alpha})} \cos \left(\frac{\bar{\varphi}+\bar{\varphi}_{0}}{2}\right) \cos \left(\frac{\bar{\varphi}-\bar{\varphi}_{0}}{2}\right)\right)\right), \\
& D_{11}^{2}\left(y_{1}, y_{3}\right)=-\frac{\mathrm{e}^{\mathrm{i} \frac{\pi}{4}} \sqrt{\sin \bar{\theta}}}{2 \beta \sqrt{2 \pi}}\left(\mathrm{e}^{-\mathrm{i} u_{R}^{2}} \cos \left(\bar{\varphi}_{0}+\frac{\bar{\varphi}+\bar{\varphi}_{0}}{2}\right) \mathrm{e}^{\mathrm{i} \frac{k \cos \bar{\gamma}}{\beta} y_{3}}\right.
\end{aligned}
$$

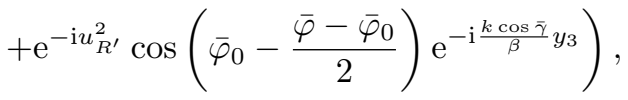

$$
\begin{aligned}
& D_{33}^{0}\left(y_{1}, y_{3}\right)=-\left(\frac{\cos \bar{\gamma}}{\beta}\right)^{2}\left(E\left(u_{R}\right) \mathrm{e}^{\mathrm{i} \frac{k}{\beta} \cos \bar{\gamma} y_{3}}+E\left(u_{R^{\prime}}\right) \mathrm{e}^{-\mathrm{i} \frac{k}{\beta} \cos \bar{\gamma} y_{3}}\right) \\
& D_{33}^{1}\left(y_{1}, y_{3}\right)=\frac{2 \mathrm{i} \cos \bar{\gamma} \mathrm{e}^{\mathrm{i} \frac{\pi}{4}} \sqrt{\sin \bar{\theta}}}{\beta \sqrt{2 \pi}}\left(\mathrm{e}^{-\mathrm{i} u_{R}^{2}} \sin \frac{\bar{\varphi}+\bar{\varphi}_{0}}{2} \mathrm{e}^{\mathrm{i} \frac{k \cos \bar{\gamma}}{\beta}} y_{3}\right. \\
& \left(1-\frac{\sin \bar{\theta}}{\cos \bar{\gamma}} \cos \left(\frac{\bar{\varphi}-\bar{\varphi}_{0}}{2}\right) \sin \left(\frac{\bar{\varphi}+\bar{\varphi}_{0}}{2}\right)\right)
\end{aligned}
$$

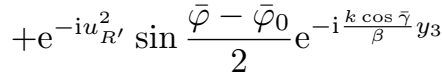

$$
\begin{aligned}
& \left.\left(1-\frac{\sin \bar{\theta}}{\cos \bar{\gamma}} \cos \left(\frac{\bar{\varphi}+\bar{\varphi}_{0}}{2}\right) \sin \left(\frac{\bar{\varphi}-\bar{\varphi}_{0}}{2}\right)\right)\right), \\
& D_{33}^{2}\left(y_{1}, y_{3}\right)=\frac{\beta \mathrm{e}^{\mathrm{i} \frac{\pi}{4}} \sqrt{\sin \bar{\theta}}}{2 \sqrt{2 \pi}}\left(\mathrm{e}^{-\mathrm{i} u_{R}^{2}} \cos \left(\bar{\varphi}_{0}+\frac{\bar{\varphi}+\bar{\varphi}_{0}}{2}\right) \mathrm{e}^{\mathrm{i} \frac{k \cos \bar{\gamma}}{\beta} y_{3}}\right.
\end{aligned}
$$

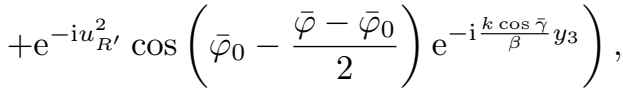




$$
\begin{aligned}
& D_{13}^{0}\left(y_{1}, y_{3}\right)=-\left(\frac{(-M+\cos \bar{\alpha}) \cos \bar{\gamma}}{\beta^{3}}\right)\left(E\left(u_{R}\right) \mathrm{e}^{\mathrm{i} \frac{k}{\beta} \cos \bar{\gamma} y_{3}}-E\left(u_{R^{\prime}}\right) \mathrm{e}^{-\mathrm{i} \frac{k}{\beta} \cos \bar{\gamma} y_{3}}\right), \\
& D_{13}^{1}\left(y_{1}, y_{3}\right)=\frac{2 \mathrm{i}(-M+\cos \bar{\alpha}) \mathrm{e}^{\mathrm{i} \frac{\pi}{4}} \sqrt{\sin \bar{\theta}}}{\beta^{2} \sqrt{2 \pi}} \\
& \left(\mathrm { e } ^ { - \mathrm { i } u _ { R } ^ { 2 } } \mathrm { e } ^ { \mathrm { i } \frac { k \operatorname { c o s } \overline { \gamma } } { \beta } y _ { 3 } } \left(\frac{1}{2} \sin \frac{\bar{\varphi}+\bar{\varphi}_{0}}{2}-\frac{\cos \bar{\gamma}}{2(-M+\cos \bar{\alpha})} \cos \frac{\bar{\varphi}+\bar{\varphi}_{0}}{2}\right.\right. \\
& \left.+\frac{\sin \bar{\theta}}{-M+\cos \bar{\alpha}} \cos \left(\frac{\bar{\varphi}-\bar{\varphi}_{0}}{2}\right) \sin \left(\frac{\bar{\varphi}+\bar{\varphi}_{0}}{2}\right) \cos \left(\frac{\bar{\varphi}+\bar{\varphi}_{0}}{2}\right)\right) \\
& +\mathrm{e}^{-\mathrm{i} u_{R^{\prime}}^{2}} \mathrm{e}^{-\mathrm{i} \frac{k \cos \bar{\gamma}}{\beta}} y_{3}\left(-\frac{1}{2} \sin \frac{\bar{\varphi}-\bar{\varphi}_{0}}{2}+\frac{\cos \bar{\gamma}}{2(-M+\cos \bar{\alpha})} \cos \frac{\bar{\varphi}-\bar{\varphi}_{0}}{2}\right. \\
& \left.\left.-\frac{\sin \bar{\theta}}{-M+\cos \bar{\alpha}} \cos \left(\frac{\bar{\varphi}+\bar{\varphi}_{0}}{2}\right) \sin \left(\frac{\bar{\varphi}-\bar{\varphi}_{0}}{2}\right) \cos \left(\frac{\bar{\varphi}-\bar{\varphi}_{0}}{2}\right)\right)\right) \text {, } \\
& D_{13}^{2}\left(y_{1}, y_{3}\right)=\frac{\mathrm{e}^{\mathrm{i} \frac{\pi}{4}} \sqrt{\sin \bar{\theta}}}{2 \sqrt{2 \pi}}\left(\mathrm{e}^{-\mathrm{i} u_{R}^{2}} \sin \left(\bar{\varphi}_{0}+\frac{\bar{\varphi}+\bar{\varphi}_{0}}{2}\right) \mathrm{e}^{\mathrm{i} \frac{k \cos \bar{\gamma}}{\beta} y_{3}}+\right.
\end{aligned}
$$

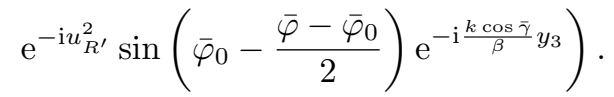

\section{REFERENCES}

Amiet, R. K. 1975 Acoustic radiation from an airfoil in a turbulent stream. Journal of Sound and Vibration 41 (4), 407-420.

Амiet, R. K. 1976 a High frequency thin-airfoil theory for subsonic flow. AIAA Journal 14 (8), 1076-1082.

Амiet, R. K. $1976 b$ Noise due to turbulent flow past a trailing edge. Journal of Sound and Vibration 47 (3), 387-393.

Амiet, R. K. 1978 Effect of the incident surface pressure field on noise due to turbulent flow past a trailing edge. Journal of Sound and Vibration 57, 305-306.

ANSYS 2015 Fluent theory guide release 16.0. ANSYS, Inc., Southpointe, 2600 ANSYS Drive, Canonsburg, PA 15317.

Arndt, R., Long, D. F. \& Glauser, M. N. 1997 The proper orthogonal decomposition of pressure fluctuations surrounding a turbulent jet. Journal of Fluid Mechanics 340, 1-33.

Bailly, C., CAndel, S. \& Lafon, P. 1996 Prediction of supersonic jet noise from a statistical acoustic model and a compressible turbulence closure. Journal of Sound and Vibration 194, 219-242.

Bailly, C., LAfon, P. \& CAndel, S. 1994 Computation of subsonic and supersonic jet mixing noise using a modified $k-\epsilon$ model for compressible free shear flows. Acta Acustica 2, 101-112.

Bechara, W., Lafon, P., Bailly, C. \& Candel, S. M. 1995 Application of a $k-\epsilon$ turbulence model to the prediction of noise for simple and coaxial jets. Journal of Acoustical Society of America 97, 3518-3513.

Bhat, T. R. S. \& Blackner, A. M. 1998 Installed jet noise prediction model for coaxial jets. In Proceedings of 9th AIAA/CEAS Aeroacoustics Conference. American Institute of Aeronautics and Astronautics, AIAA 98-79.

Bondarenko, M., Hu, Z. \& Zhang, X. 2012 Large-eddy simulation of the interaction of a jet with a wing. In Proceedings of Proceeding of 18th AIAA/CEAS Aeroacoustics Conference. American Institute of Aeronautics and Astronautics, AIAA 2012-2254.

Bridges, J. \& Wernet, M. P. 2010 Establishing consensus turbulence statistics for hot subsonic jets. In Proceedings of 16th AIAA/CEAS Aeroacoustic Conference. American Institute of Aeroacoustic and Astronautics, AIAA 2010-3751.

Brown, C. A. 2013 Jet-surface interaction test: far-field noise results. Journal of Engineering for Gas Turbines and Power 135 (7), 71201.

Bushell, K. 1975 Measurement and prediction of jet noise in flight. In Proceedings of 2nd 
Aeroacoustics Conference. American Institute of Aeronautics and Astronautics, AIAA 75461.

Bychkov, O. P. \& Faranosov, G. A. 2014 On the possible mechanism of the jet noise intensification near a wing. Acoustical Physics 60 (6), 633-646.

Curle, N. 1955 The influence of solid boundaries upon aerodynamic sound. Proceedings of the Royal Society A: Mathematical, Physical and Engineering Sciences 231, 505-514.

Fisher, M. J., HARper-Bourne, M. \& GLeGG, S. A. L. 1977 Jet engine noise source location: The polar correlation technique. Journal of Sound and Vibration 51, 23-54.

Goldstein, M. E. 2003 A generalized acoustic analogy. Journal of Fluid Mechanics 488, 315333.

Gradshteyn, I. S. \& Ryzhik, I. M. 2007 Table of integrals, series, and products, 7th edn. 30 Corporate Drive, Suite 400, Burlington, MA 01803, USA: Elsevier Academic Press.

Gudmundsson, K. \& Colonius, T. 2011 Instability wave models for the near-field fluctuations of turbulent jets. Journal of Fluid Mechanics 689, 97-128.

HeAd, R. \& FisheR, M. 1976 Jet/surface interaction noise - analysis of farfield low frequency augmentations of jet noise due to the presence of a solid shield. In Proceedings of 3rd Aeroacoustics Conference. American Institute of Aeronautics and Astronautics, AIAA 76502.

JAMESON, A. 2008 Formulation of kinetic energy preserving conservative schemes for gas dynamics and direct numerical simulation of one- dimensional viscous compressible flow in a shock tube using entropy and kinetic energy preserving schemes. Journal of Scientific Computing 34 (2), 188-208.

Jordan, P. \& Colonius, T. 2013 Wave packets and turbulent jet noise. Annual Review of Fluid Mechanis 45, 173-195.

Karabasov, S. A., Afsar, M. Z., Hynes, T. P., Dowling, A. P., McMullan, W. A., Pokora, C. D., PAge, G. J. \& McGuirk, J. J. 2010 Jet noise: acoustic analogy informed by large eddy simulation. AIAA Journal 48 (7), 1312-1325.

Kerhervé, F., Fitzpatick, J. \& Jordan, P. 2006 The frequency dependence of jet turbulence for noise source modelling. Journal of Sound and Vibration 296, 209-225.

Khavaran, A., Krejsa, E. A. \& Kim, C. M. 1994 Computation of supersonic jet mixing noise for an axisymmetric convergent-divergent nozzle. Journal of Aircraft 31 (5), 603-609.

Launder, B. E. \& Spalding, D. B. 1974 The numerical computation of turbulent flows. Computer Methods in Applied Mechanics and Engineering 3, 269-289.

Lawrence, J. L. T., Azarpevand, M. \& Self, R. H. 2011 Interaction between a flat plate and a circular subsonic jet. In proceedings of the 17th AIAA CEAS Aeroacoustic Conference. American Institute of Aeronautics and Astronautics, AIAA 2011-2754.

Lighthill, M. J. 1952 On sound generated aerodynamically. i. general theory. Proceedings of the Royal Society A: Mathematical, Physical and Engineering Sciences 211 (1107), 564-587.

Lighthill, M. J. 1954 On sound generated aerodynamically. ii. turbulence as a source of sound. Proceedings of the Royal Society A: Mathematical, Physical and Engineering Sciences 222, $1-32$.

Lilley, G. M. 1974 On the noise from jets. AGARD Noise Mechanism CP-131 13, 12.

Lyu, B., Azarpeyvand, M. \& Sinayoko, S. 2015 A trailing-edge noise model for serrated edges. In Proceeding of 21th AIAA/CEAS Aeroacoustics Conference. American Institute of Aeronautics and Astronautics, AIAA 2015-2362.

Lyu, B., Azarpeyvand, M. \& Sinayoko, S. 2016 Noise prediction for serrated trailing edges. Journal of Fluid Mechanics 793, 556-588.

Lyu, B. \& Dowling, A. P. 2016 Noise prediction for installed jets. In Proceeding of 22th AIAA/CEAS Aeroacoustics Conference. American Institute of Aeronautics and Astronautics, AIAA 2016-2986.

Macdonald, H. M. 1915 A class of diffraction problem. Proceedings of the London Mathematical Society 14, 410-427.

Mead, C. \& Strange, P. 1998 Under-wing installation effects on jet noise at sideline. In Proceedings of 4 th AIAA/CEAS Aeroacoustics Conference. American Institute of Aeronautics and Astronautics, AIAA 98-2207.

Mohan, N. K. D., Dowling, A. P., Karabasov, S. A., Xia, H., Graham, O., Hynes, 
T. P. \& Tucker, P. G. 2015 Acoustic sources and far-field noise of chevron and round jets. AIAA Journal $00(0), 1-16$.

Moore, A. 2004 A 3D prediction of the wing reflection of aero engine noise. In Proceedings of 10th AIAA/CEAS Aeroacoustics Conference. American Institute of Aeronautics and Astronautics, AIAA 2004-2865.

Naqavi, I. Z., Wang, Z-N, Tucker, P. G., Mahak, M. \& Strange, P. 2016 Far-field noise prediction for jets using Large-eddy Simulation (LES) and Ffowcs Williams-Hawkings (FW$\mathrm{H})$ method. Internal Journal of Aeroacoustics 15 (8).

Papamoschou, D. 2010 Prediction of jet noise shielding. In Proceedings of the 48th AIAA Aerospace Sciences Meeting. American Institute of Aeronautics and Astronatics, AIAA $2010-653$

Pastouchenko, N. N. \& TAm, C. K. W. 2007 Installation effects on the flow and noise of wing mounted jets. AIAA Journal 45 (12), 2851-2860.

Pepper, C. B., Nascarella, M. A. \& Kendall, R. J. 2003 A review of the effects of aircraft noise on wildlife and humans, current control mechanisms, and the need for further study. Environmental Management 32, 3024.

Piantanida, S., Jaunet, V., Huber, J., Wolf, W., Jordan, P. \& Cavalieri, A. V. G. 2015 Scattering of turbulent-jet wavepackets by a swept trailing edge. In Proceedings of 21st AIAA/CEAS Aeroacoustic Conference. American Institute of Aeroacoustic and Astronautics, AIAA 2015-2998.

Roger, M. \& Moreau, S. 2005 Back-scattering correction and further extensions of Amiet's trailing-edge noise model. part 1: theory. Journal of Sound and Vibration 286 (1-2), 477506.

Sengupta, G. 1983 Analysis of jet-airframe interaction noise. In Proceedings of 8th Aeroacoustics Conference. American Institute of Aeronautics and Astronautics, AIAA 83-783.

SHEARIN, J. G. 1983 Investigation of jet-installation noise sources under static conditions. NASA Technical Report 2181. NASA Langley Research Center, , Hampton, Virgina.

Stevens, R., Bryce, W. \& Szewczyk, V. 1983 Model and full-scale studies of the exhaust noise from a bypass engine in flight. In Proceedings of 8th Aeroacoustics Conference. American Institute of Aeronautics and Astronautics, AIAA 83-751.

SzewczyK, V. 1979 Coaxial jet noise in flight. In Proceedings of 5th Aeroacoustics Conference. American Institute of Aeronautics and Astronautics, AIAA 79-636.

TAM, C. K. W. \& Auriault, L. 1999 Jet mixing noise from fine-scale turbulence. AIAA Journal 37 (2), 145-153.

Tinney, C. E., Glauser, M. N. \& Ukeiley, L. S. 2008 a Low-dimensional characteristics of a transonic jet. part 1. proper orthogonal decomposition. Journal of Fluid Mechanics 612, $107-141$.

Tinney, C. E. \& Jordan, P. 2008 The near pressure field of co-axial subsonic jets. Journal of Fluid Mechanics 611, 175-204.

Tinney, C. E., Ukeiley, L. S. \& Glauser, M. N. $2008 b$ Low-dimensional characteristics of a transonic jet. part 2. estimate and far-field prediction. Journal of Fluid Mechanics 615, $53-92$.

Vera, J., Self, R. H. \& Kingan, M. J. 2015 The prediction of the radiated pressure spectrum produced by jet-wing interaction. In Proceedings of 21st AIAA/CEAS Aeroacoustic Conference. American Institute of Aeroacoustic and Astronautics, AIAA 2015-2216.

WAng, M. 1981 Wing effect on jet noise propagation. Journal of Aircraft 18, 295-302.

WAy, D. \& Turner, B. 1980 Model tests demonstrating under-wing installation effects on engine exhaust noise. In Proceedings of 6th Aeroacoustics Conference. American Institute of Aeronautics and Astronautics, AIAA 80-1048.

White, F. M. 2005 Fluid Mechanics, 5th edn. McGraw-Hill.

Williams, J. E. F. 1963 The noise from turbulence convected at high speed. Philosophical Transactions of the Royal Society A: Mathematical, Physical and Engineering Sciences 255, 469-503.

Williams, J. E. F. \& Hall, L. H. 1970 Aerodynamic sound generation by turbulent flow in the vicinity of a scattering half plane. Journal of Fluid Mechanics 40, 657-670. 